TRANSACTIONS OF THE

AMERICAN MATHEMATICAL SOCIETY

Volume 355, Number 8, Pages 3181-3208

S 0002-9947(03)03293-8

Article electronically published on April 11, 2003

\title{
ON THE CLIFFORD ALGEBRA OF A BINARY FORM
}

\author{
RAJESH S. KULKARNI
}

\begin{abstract}
The Clifford algebra $C_{f}$ of a binary form $f$ of degree $d$ is the $k$ algebra $k\{x, y\} / I$, where $I$ is the ideal generated by $\left\{(\alpha x+\beta y)^{d}-f(\alpha, \beta) \mid\right.$ $\alpha, \beta \in k\} . C_{f}$ has a natural homomorphic image $A_{f}$ that is a rank $d^{2}$ Azumaya algebra over its center. We prove that the center is isomorphic to the coordinate ring of the complement of an explicit $\Theta$-divisor in $\mathrm{Pic}_{C / k}^{d+g-1}$, where $C$ is the curve $\left(w^{d}-f(u, v)\right)$ and $g$ is the genus of $C$.
\end{abstract}

\section{INTRODUCTION}

Let $f$ be a form of degree $d$ in $n$ variables over a field $k$. Then the Clifford algebra $C_{f}$ is the $k$-algebra $k\left\{x_{1}, \ldots, x_{n}\right\} / I$ where $k\left\{x_{1}, \ldots, x_{n}\right\}$ is the free associative algebra in $n$ variables and $I$ is the ideal generated by $\left\{\left(\alpha_{1} x_{1}+\ldots+\alpha_{n} x_{n}\right)^{d}-\right.$ $\left.f\left(\alpha_{1}, \ldots, \alpha_{n}\right) \mid \alpha_{1}, \ldots, \alpha_{n} \in k\right\}$. If $d=2$, this is the classical Clifford algebra of a quadratic form. If $d>2$, then this is sometimes called the generalized Clifford algebra and has been studied by various authors, including Roby [21], Revoy [20], and Childs [3].

The first case of higher degree (that is, $d=3, n=2$ ) was studied by Haile in 8 and 9 . He showed that the Clifford algebra of a binary cubic form over a field $k$, with characteristic $\neq 2,3$, is Azumaya with center the affine coordinate ring of an elliptic curve. Further, this elliptic curve is the Jacobian of the projective curve given by the equation $\left(w^{3}-f(u, v)\right)$.

The case of $d>3$ displays different behavior. Namely, using results of [20], it is easy to see that $C_{f}$ contains a free algebra on two variables. In particular, $C_{f}$ is not finitely generated over its center (as a module) and hence is not Azumaya. However, it has been shown ([10]) that the dimension of any representation is divisible by $d$. Furthermore, $\tilde{C}_{f}=C_{f} /(\bigcap$ ker $\eta)$ is Azumaya over its center, where the intersection is taken over all the kernels of dimension $d$ representations. The question of describing the center is thus interesting from this point of view.

The main tool in understanding representations of the Clifford algebra (for $d \geq 3$ ) was introduced by Van den Bergh in 22. While previous results about representations $([8,[10)$ were global in nature, this idea gave a local result. To understand the statement we introduce some notation. Denote by $C$ the projective curve given by the equation $\left(w^{d}-f(u, v)\right)$. Let $g$ be the genus of this degree $d$ curve. Assume that $k$ is algebraically closed of characteristic 0 and that the form $f$ has no repeated factors over $k$. By an $r d$-dimensional representation of $C_{f}$, we mean a homomorphism from $C_{f}$ to $M_{r d}(k)$, the $r d \times r d$ matrices over $k$. Then the result

Received by the editors January 1, 2002

2000 Mathematics Subject Classification. Primary 16H05, 16G99, 14H40, 14K30. 
(22], Proposition 1 in Section 1 and Lemma 2 in Section 2.2) states that there is a one-to-one correspondence between equivalence classes of $r d$-dimensional representations of $C_{f}$ and isomorphism classes of vector bundles $\mathcal{E}$ on $C$ of rank $r$ and degree $(d+g-1)$ such that $H^{0}(C, \mathcal{E}(-1))=0$. It should be emphasized that this result is constructive in nature. Namely, for a vector bundle that satisfies the conditions of the theorem, we can construct an explicit representation of $C_{f}$ (on the global sections of $\mathcal{E}$ ), and vice versa.

This result suggests that the center of $\tilde{C_{f}}$ should be the coordinate ring of an affine open set in the (translated) Jacobian of the curve $C$. In fact, a closer inspection reveals that the open set is the complement of a $\Theta$-divisor in $\mathrm{Pic}_{C / k}^{d+g-1}$. The results of [8] and [9] pertaining to the case of binary cubic forms give credence to this belief. In this paper, we prove this result under the assumptions that $k$ is an infinite field, $f$ has no repeated factors over an algebraic closure of $k$ and $\operatorname{char}(k)$ does not divide $d$.

First consider the case when $C$ has a $k$-rational point. For this case, we briefly describe the strategy to construct the required isomorphism. Let $Z_{k}$ denote the center of $\tilde{C_{f}}$ and $A_{k}$ denote the coordinate ring of the complement of a $\Theta$-divisor in $\operatorname{Pic}_{C / k}^{d+g-1}$. We compute the graded module associated to the direct image of the universal bundle under the projection $C \times_{k}$ Spec $A_{k} \rightarrow \mathbb{P}_{k}^{1} \times_{k}$ Spec $A_{k}$. In fact, it turns out that this direct image is isomorphic to the pull-back of the direct image of the universal bundle under the projection on the second factor. This allows us to define a homomorphism $\varphi$ from $\tilde{C}_{f}$ to End $A_{k} P_{0}$, where $P_{0}$ is the $A_{k}$-module of global sections of the direct image of the universal line bundle. This morphism has the important property that any $d$-dimensional representation of $\tilde{C}_{f}$ factors through End $A_{A_{k}} P_{0}$. This implies that the morphism $\varphi$ is injective. Using the fact that $A_{k}$ is integrally closed, we prove that $\varphi$ maps $Z_{k}$ to $A_{k}$. Then our goal is to show that this morphism on the centers is an isomorphism.

In our original approach ([12]), we showed that $\varphi$ is an isomorphism by proving sufficiently many geometric properties of $\varphi$ to use the Main Theorem of Zariski. However, it was pointed out to us that a more direct approach might be to construct a morphism $\eta$ from Spec $Z_{k}$ to Spec $A_{k}$ using some of the ideas from 23. Namely, we construct a natural transformation between functors which are represented by the schemes $\operatorname{Spec} Z_{k}$ and $\operatorname{Spec} A_{k}$. This then yields the required morphism. It is then easy to show that the composition $\eta \circ \varphi:$ Spec $A_{k} \rightarrow$ Spec $A_{k}$ is the identity morphism. Using the injectivity of $\varphi: Z_{k} \rightarrow A_{k}$, we see that this is an isomorphism.

Now we discuss the case when $C$ does not have a $k$-rational point. In this case, there exists a finite Galois extension $k^{\prime} / k$ such that $C\left(k^{\prime}\right)$ is nonempty. The results discussed in the previous paragraphs show that after this base extension, the center $Z_{k^{\prime}}$ is isomorphic (via $\varphi$ ) to $A_{k^{\prime}}$, the coordinate ring of the complement of the $\Theta$ divisor in $\operatorname{Pic}_{C^{\prime} / k^{\prime}}^{d+g-1}$. We show that this morphism descends to $k$. For any element $\sigma \in \operatorname{Gal}\left(k^{\prime} / k\right)$, consider the automorphism $\mu=\sigma^{-1} \circ \varphi^{-1} \circ \sigma \circ \varphi$. Using a calculation of the universal bundle $\mathcal{P}$, we show that the restriction of its pull-back under $\mu$ to a closed point $y$ is isomorphic to $\mathcal{P}_{y}$. This then shows that $\mu$ is the identity morphism, giving us the required descent.

The paper is organized in six sections. In the second section, we review some basic material about Jacobians of curves, keeping in mind the audience for this paper. In Section 3, we study the restriction of the direct image of the universal 
line bundle on the complement of the $\Theta$-divisor. In Section 4 , the morphism $\varphi$ is constructed and its properties are studied. In Section 5, we construct the morphism $\eta$. In the last section, the main theorem is proved, identifying the center of $\tilde{C}_{f}$.

In the following, we assume that the binary form $f$ has no repeated factors over an algebraic closure of $k$ and that the characteristic of $k$ does not divide $d$.

\section{Notation And Conventions}

- All rings have an identity element.

- Let $B$ be an $A$-algebra and $X$ be a Spec $A$-scheme. We denote $X \times_{\text {Spec } A}$ Spec $B$ by $X \times{ }_{A} B$.

- All schemes are locally Noetherian and all morphisms are of locally finite type.

- $(\mathrm{Sch} / k)$ denotes the category of schemes over Spec $k$ whose structure morphisms are locally of finite type. Also $(\mathrm{Sch} / X)_{\mathrm{fl}}$ denotes the flat site on $X$. All sheafifications are with respect to the flat topology.

- $($ fppf $)=$ faithfully-flat and of finite presentation.

- The projections of fibred products onto the $i^{\text {th }}$ component $X_{i}$ are denoted by either $p_{i}, \pi_{i}$ or $p_{X_{i}}, \pi_{X_{i}}$.

- For any scheme $X$ and a positive integer $n, X^{(n)}$ denotes the $n$-fold symmetric product of $X$.

- The terms line bundles (respectively vector bundles) and invertible sheaves (respectively locally free sheaves) are used interchangeably.

- For any vector bundle $\mathcal{E}, \chi(\mathcal{E})$ denotes the Euler characteristic of $\mathcal{E}$.

- The translation morphism induced by an element $a$ of an abelian variety $A$ is denoted by $t_{a}$.

- All the fields are assumed to be infinite.

\section{The universal Property of the PiCARD SCHEME AND THE UNIVERSAL INVERTIBLE SHEAF}

In this section, we recall some basic properties of Picard schemes of curves and the universal invertible sheaf associated with them. The general references for this section are [2], Chapter 8 , and [15].

Let $S$ be a base scheme, $X$ an $S$-scheme and $f: X \rightarrow S$ be the structure morphism. For any $S$-scheme $T$, let $q$ denote the projection $X \times_{S} T \rightarrow T$. The functor $\operatorname{Pic}_{X / S}$ which associates to any $S$-scheme $T$ the Picard group $\operatorname{Pic}\left(X \times_{S} T\right)$ is called the Picard functor. This functor is not representable, since it is not a sheaf even with respect to the Zariski topology. We consider its sheafification with respect to the (fppf)-topology (called the relative Picard functor). The sheafified functor is representable by a scheme which is also denoted by $\mathrm{Pic}_{X / S}$. If $f$ has a section, the sheafified functor is isomorphic to the functor that assigns to any $S$-scheme $T$ the group $\operatorname{Pic}\left(X \times{ }_{S} T\right) / q^{*} \operatorname{Pic}(T)$ (Proposition 4 in [2], Chapter 8).

There is another description for the relative Picard functor for more restricted situations ([2], Chapter 8). We assume that $f_{*}\left(\mathcal{O}_{X}\right)=\mathcal{O}_{S}$ holds universally (that is, this formula holds true after any base change) and that $f$ admits a section $\varepsilon: S \rightarrow X$. For a line bundle $\mathcal{L}$ on $X$, an isomorphism $\alpha: \mathcal{O}_{S} \stackrel{\sim}{\rightarrow} \varepsilon^{*}(\mathcal{L})$ is called a rigidification of $\mathcal{L}$. The pair $(\mathcal{L}, \alpha)$ is referred to as a rigidified line bundle along the section $\varepsilon$. There is an obvious notion of morphisms of rigidified line bundles. Then we consider the functor $(P, \varepsilon):(\mathrm{Sch} / S)^{0} \rightarrow(\mathrm{Sets})$ which associates to an 
$S$-scheme $T$ the set of isomorphism classes of line bundles on $X_{T}=X \times{ }_{S} T$ that are rigidified along the induced section $\varepsilon_{T}: T \rightarrow X_{T}$. The functor $(P, \varepsilon)$ is canonically isomorphic to the relative Picard functor $\mathrm{Pic}_{X / S}$.

Now consider a smooth projective curve $C$ over a field $k$ of genus $g$. We call the open (and closed) subfunctor that considers only invertible sheaves $\mathcal{L}$ on $C \times_{k} T$ whose restriction to $C \times\{t\}$ is of fixed degree $n$ for any point $t$ the relative Picard functor of degree $n$. The scheme that represents it is denoted by $\mathrm{Pic}_{C / k}^{n}$. This is a smooth, projective scheme over $k$.

Next suppose the curve $C$ has a $k$-rational point. Fix a section $\varepsilon: S p e c k \rightarrow C$ corresponding to such a rational point. Let $\mathcal{O}(1)$ be a very ample line bundle on $C$ of degree $d$. Then the scheme $\mathrm{Pic}_{C / k}$ (respectively $\mathrm{Pic}_{C / k}^{d+g-1}$ ) also represents $(P, \varepsilon)$ (respectively $\left(P^{d+g-1}, \varepsilon\right)$, which is an open subfunctor of $(P, \varepsilon)$ consisting of rigidified line bundles of degree $(d+g-1)$ ). So the identity on $\mathrm{Pic}_{C / k}$ (respectively $\operatorname{Pic}_{C / k}^{d+g-1}$ ) gives a line bundle $\mathcal{P}$ (respectively $\mathcal{P}$ ) on $C \times_{k} \mathrm{Pic}_{C / k}$ (respectively $C \times{ }_{k} \mathrm{Pic}_{C / k}^{d+g-1}$ ) that is canonically rigidified along the induced section. The sheaf $\mathcal{P}$ is called the universal (or Poincaré) line bundle for $(C / k, \varepsilon)$. In fact, it is easy to see that the corresponding universal line bundle on $C \times{ }_{k} \mathrm{Pic}_{C / k}^{d+g-1}$ is the pull-back of the universal line bundle on $C \times{ }_{k} \mathrm{Pic}_{C / k}$ under the canonical inclusion. The next proposition, which is adapted from [2], Chapter 8, 8.4, Proposition 4, justifies this terminology.

Proposition 2.1. With the notation as above, the functor $(P, \varepsilon)$ is representable by a scheme which we denote by $\mathrm{Pic}_{C / k}$. The universal line bundle $\mathcal{P}$ has the following property: For any $k$-scheme $X$, and for any line bundle $\mathcal{L}$ on $C \times_{k} X$ that is rigidified along the induced section $\varepsilon_{X}$, there exists a unique morphism $g: X \rightarrow \operatorname{Pic}_{C / k}$ such that $\mathcal{L}$, as a rigidified line bundle, is isomorphic to the pull-back of $\mathcal{P}$ under the morphism $\operatorname{id}_{C} \times g$. A similar statement is true for $\left(P^{d+g-1}, \varepsilon\right)$ if the line bundle $\left.\mathcal{L}\right|_{C \times_{k}\{x\}}$ is of degree $(d+g-1)$ for any point $x \in X$.

Next we want to relate the schemes representing the relative Picard functors corresponding to $C / k$ and $C_{K} / K$. Here $K$ is an arbitrary field extension of $k$ and $C_{K}=C \times_{k} K$. The next proposition is true for arbitrary base extensions as well. See [7, Section 3.

Let $\pi: C \rightarrow$ Spec $k$ be the structure morphism. Let $K$ be any field extension of $k$ and $f:$ Spec $K \rightarrow$ Spec $k$ be the corresponding base extension. We continue to use the same notation as above in the following proposition.

Proposition 2.2. The Picard scheme $\mathrm{Pic}_{C_{K} / K}$ is isomorphic to $\mathrm{Pic}_{C / k} \times_{k} K$ as a K-scheme. Let $g: \mathrm{Pic}_{C_{K} / K} \rightarrow \mathrm{Pic}_{C / k} \times_{k} K$ be such an isomorphism, and let $\mathcal{P}$ be the universal line bundle for $(C / k, \varepsilon)$. Then $h_{1}^{*} h_{2}^{*}(\mathrm{id} \times g)^{*}\left(p_{1} \times \mathrm{id}\right)^{*} \mathcal{P}$ is the universal line bundle for $\left(C_{K} / K, f \circ \varepsilon\right)$, where the morphisms are described in the following sequence:

$$
C_{K} \times_{K} \mathrm{Pic}_{C_{K} / K} \stackrel{h_{1}}{\longrightarrow} C \times_{k} K \times_{K} \mathrm{Pic}_{C_{K} / K} \stackrel{h_{2}}{\longrightarrow} C \times_{k} K \times_{k} \mathrm{Pic}_{C / k} .
$$

Remark 2.3. The first part of the above proposition remains true even if the curve $C / k$ does not have a $k$-rational point. Namely, the formation of the Picard scheme is compatible with the base change. This follows because, in fact, the usual construction of the Picard scheme is to construct it after a base extension so that the curve has a rational point (over the extended field), and then to use descent. For 
example, see [15]. Also, the above proposition remains true if we restrict to the open subfunctor of degree $(d+g-1)$. This is clear since the degree of a line bundle does not change under a pull-back via base extension of fields.

Remark 2.4. We have considered in the above discussion only the open subfunctors corresponding to degree $(d+g-1)$. But all the facts about representability remain true for any degree. The facts about the universal line bundle also remain true as long as the curve under consideration has a rational point over its base field. The proofs of these assertions are identical to the case of degree $(d+g-1)$.

The Picard scheme is a group scheme (variety) over the base field. The Picard scheme of degree 0 is the Jacobian variety of $C / k$ and is also denoted by $J$. The group operation corresponds to tensoring of line bundles. We define specific $\Theta-$ divisors in Picard schemes of degrees $(g-1)$ and $(d+g-1)$.

Definition 2.5. Let $C / k$ be a curve with the hypothesis as above (we do not need $C$ to have a $k$-rational point). The $\Theta$-divisor (of degree $(g-1))$ is the schematic image of the canonical morphism

$$
(C)^{(g-1)} \rightarrow \mathrm{Pic}_{C / k}^{g-1}, \quad D_{T} \mapsto\left[D_{T}\right],
$$

where, for any $k$-scheme $T$ and for any $T$-valued point $D_{T}$ of $(C)^{(g-1)},\left[D_{T}\right]$ denotes the element of $\mathrm{Pic}_{C / k}^{g-1}$ corresponding to $D_{T}$.

Recall that the curve $C$ is equipped with a (very ample) line bundle $\mathcal{O}(1)$ of degree $d$. This gives a canonically defined $\Theta$-divisor in $\mathrm{Pic}_{C / k}^{d+g-1}$, as the image of the $\Theta$-divisor under the morphism

$$
\mathrm{Pic}_{C / k}^{g-1} \stackrel{\otimes \mathcal{O}(1)}{\longrightarrow} \mathrm{Pic}_{C / k}^{d+g-1} .
$$

Now let $K$ be a field extension of $k$. By considering the commutative diagram

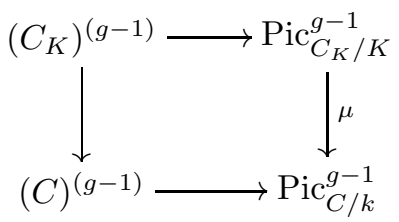

we see that the pull-back of the $\Theta$-divisor under the morphism $\mu$ is the $\Theta$-divisor for $C_{K} / K$. In fact, the same is true as well for the complement of the $\Theta$-divisor.

Proposition 2.6. Let $C / k$ be a curve as before, and let $K$ be a field extension of $k$. Let $\mathcal{L}$ be a rigidified line bundle on $C_{K}$ of degree $(d+g-1)$. Then the image of the unique morphism Spec $K \stackrel{i}{\rightarrow} \operatorname{Pic}_{C / k}^{d+g-1}$ corresponding to $\mathcal{L}$ lies in the complement of the $\Theta$-divisor if and only if $h^{0}\left(C_{K}, \mathcal{L}(-1)\right)=0$.

Proof. One direction is immediate once we note that the fibre over a closed point $x$ under the composite morphism

$$
C^{(g-1)} \rightarrow \operatorname{Pic}_{C / k}^{g-1} \rightarrow \operatorname{Pic}_{C / k}^{d+g-1}
$$

either is empty or is exactly the linear system $H^{0}\left(C \times_{k} k(x), \mathcal{L}_{x}(-1)\right)$, where $\mathcal{L}_{x}$ is the line bundle corresponding to the point $x$ in $\operatorname{Pic}_{C / k}^{d+g-1}$. This idea can be formalized by using the Poincaré bundle. 
For the other direction, if $h^{0}\left(C_{K}, \mathcal{L}(-1)\right) \neq 0$, then we may choose a global section $s$ of $\mathcal{L}(-1)$. Then the divisor $D_{s}$ of this section gives an element of $C^{(g-1)}$. Using the canonical morphism, this gives an invertible sheaf $\mathcal{L}_{2}$ on $C_{K}$ that has a global section whose divisor is $D_{s}$. Furthermore, the image of Spec $K$ corresponding to $\mathcal{L}_{2}$ is by definition in the $\Theta$-divisor. But since their global sections have the same divisors, the invertible sheaves $\mathcal{L}(-1)$ and $\mathcal{L}_{2}$ must be isomorphic. This gives the other direction.

We record an easy corollary for future reference.

Corollary 2.7. With the hypothesis as above, the pull-back (or the inverse image) of the complement of the $\Theta$-divisor in $\mathrm{Pic}_{C / k}^{d+g-1}$ under the canonical morphism

$$
\operatorname{Pic}_{C_{K} / K}^{d+g-1} \longrightarrow \operatorname{Pic}_{C / k}^{d+g-1}
$$

is the complement of the $\Theta$-divisor in $\operatorname{Pic}_{C_{K} / K}^{d+g-1}$.

Remark 2.8. Note that our idea of the proof of the corollary depends on $C$ having a $k$-rational point. However, by Remark 2.3 the same question can be asked if $C$ does not have a $k$-rational point. We consider a finite Galois extension $k^{\prime} / k$ (with Galois group $G$ ) so that $C\left(k^{\prime}\right)$ is nonempty. Let $C^{\prime}=C \times_{k} k^{\prime}$. Then $\operatorname{Pic}_{C^{\prime} / k^{\prime}}^{d+g-1}$ has a $G$-action and gives, by descent, $\mathrm{Pic}_{C / k}^{d+g-1}$. The morphism

$$
\left(C^{\prime}\right)^{(g-1)} \stackrel{h_{k^{\prime}}}{\longrightarrow} \operatorname{Pic}_{C^{\prime} / k^{\prime}}^{d+g-1}
$$

is $G$-equivariant, and so the image of $h_{k^{\prime}}$ is $G$-invariant, which descends to the $\Theta$ divisor in $\operatorname{Pic}_{C / k}^{d+g-1}$. Hence the complement of the $\Theta$-divisor in $\mathrm{Pic}_{C^{\prime} / k^{\prime}}^{d+g-1}$ descends to the complement of the $\Theta$-divisor in $\mathrm{Pic}_{C / k}^{d+g-1}$.

2.1. The universal line bundle on $C \times{ }_{k^{\prime}} \mathbf{P i c}_{C^{\prime} / k^{\prime}}^{d+g-1}$. In this section, we would like to explicitly construct the universal line bundle on $C \times{ }_{k^{\prime}} \mathrm{Pic}_{C^{\prime} / k^{\prime}}^{d+g-1}$. Though we consider only curves of degree $d$ in $\mathbb{P}^{2}$, most of the calculations remain valid for arbitrary curves. Our goal is to find a formula which relates the universal line bundle to its pull-back under a Galois automorphism of the base field.

We start by recalling the construction of the universal line bundle for degree 0 . Let $C$ be a smooth curve over a field $k$ such that $C^{\prime}=C \times_{k} k^{\prime}$ has a $k^{\prime}$-rational point. We assume that $k^{\prime} / k$ is a finite Galois extension. In our case, this follows from the assumption that the characteristic of $k$ does not divide $d$. To see this, note that the curve given by $\left(w^{d}-f(u, v)\right)$ has a rational point over the splitting field of the polynomial $x^{d}-f(a, b)$ for any elements $a, b \in k$. Let $J$ denote the Jacobian variety $\mathrm{Pic}_{C^{\prime} / k^{\prime}}^{0}$. Fix a rational point $P$ on $C^{\prime}$. Denote by $\Theta_{0}$ the theta divisor in $J$ obtained by translation of the usual $\Theta$-divisor in $\operatorname{Pic}_{C^{\prime} / k^{\prime}}^{g-1}$ by $\mathcal{L}([-(g-1) P])$. This is the same as the divisor of $\mathcal{L}(\Theta)(-1) \otimes \mathcal{L}(-(g-1) P)$, where $\Theta$ is the theta divisor in $\operatorname{Pic}_{C^{\prime} / k^{\prime}}^{d+g-1}$ defined earlier. We have the morphism ([15, Section 2)

$$
h: C^{\prime} \longrightarrow J,
$$

which maps a closed point $Q$ to $\mathcal{L}([Q-P])$. For any divisor $D$ in the Jacobian variety, we denote by $\mathcal{L}^{\prime}(D)$ the line bundle $m^{*} \mathcal{L}(D) \otimes p^{*} \mathcal{L}(D)^{-1} \otimes q^{*} \mathcal{L}(D)^{-1}$ on $J \times{ }_{k^{\prime}} J$, where $m$ is the multiplication morphism on $J$ and $p, q$ are the projections onto the first and second factors, respectively. Finally, write $\Theta_{0}^{-}$for the image of 
$\Theta_{0}$ under the morphism $(-1)_{J}: J \rightarrow J$, and $\left(\Theta_{0}\right)_{a}$ for $t_{a} \Theta_{0}=\Theta_{0}+a$. Denote $\left(\Theta_{0}^{-}\right)_{a}$ by $\left(\Theta_{0}\right)_{a}^{-}$.

With this notation, Lemma 6.8 of [15] says the following:

Lemma 2.9. The sheaf $\mathcal{L}=\left(h \times(-1)_{J}\right)^{*} \mathcal{L}^{\prime}\left(\Theta_{0}^{-}\right)$is isomorphic to the universal invertible sheaf given by the universal property of $J$.

Using the line bundle of the last lemma, we construct the universal line bundle for $C^{\prime} \times{ }_{k^{\prime}} \mathrm{Pic}_{C^{\prime} / k^{\prime}}^{d+g-1}$. This construction is well known; see, for example, 1], Chapter 4, Lemma 2.2 and the discussion preceding it. Denote by $L_{0}$ the line bundle

$$
\mathcal{L}(-(g-1) P)(-1)
$$

on $C^{\prime}$, and by $p_{1}$ the projection of $C^{\prime} \times_{k^{\prime}} J$ onto the first factor. The morphism $\eta$ is defined as

$$
\begin{array}{lll}
\operatorname{Pic}_{C^{\prime} / k^{\prime}}^{d+g-1} & \stackrel{\eta}{\rightarrow} & J \\
L & \mapsto & L \otimes L_{0} .
\end{array}
$$

We can now state the lemma describing the universal bundle of degree $(d+g-1)$.

Lemma 2.10. With the above notation, the line bundle $\mathcal{P}=\left(\operatorname{id}_{C^{\prime}} \times \eta\right)^{*} \mathcal{L} \otimes p_{1}^{*}\left(L_{0}^{-1}\right)$ is the universal line bundle of degree $(d+g-1)$.

Proof. Let $\varepsilon$ denote the section of $C^{\prime} \rightarrow k^{\prime}$ and let $\varepsilon_{\mathrm{Pic}_{C^{\prime} / k^{\prime}}^{d+g-1}}, \varepsilon_{J}$ denote the induced sections. Then it follows that

$$
\left(\operatorname{id}_{C^{\prime}} \times \eta\right) \circ \varepsilon_{\operatorname{Pic}_{C^{\prime} / k^{\prime}}^{d+g-1}}=\varepsilon_{J} \circ \eta .
$$

This means that $\left(\operatorname{id}_{C^{\prime}} \times \eta\right)^{*} \mathcal{L}$ is rigidifiable. Also, $p_{1}^{*}\left(L_{0}^{-1}\right)$ is rigidifiable along $\varepsilon_{\text {Pic }_{C / k}^{d+g-1}}$. Thus $\mathcal{P}$ is a rigidifiable line bundle. By Remark 2.15, this is sufficient, and we may make any choice of rigidification.

Now let $T$ be any $k^{\prime}$-scheme and let $\mathcal{L}_{T}$ be a rigidified line bundle on $C^{\prime} \times{ }_{k^{\prime}} T$ such that for any $t \in T$, the restriction $\left(\mathcal{L}_{T}\right)_{t}$ is of degree $(d+g-1)$. Then $\mathcal{L}_{T}^{\prime}=\mathcal{L}_{T} \otimes p_{1}^{*}\left(L_{0}^{-1}\right)$ is a rigidified line bundle on $C^{\prime} \times_{k^{\prime}} T$ such that its restriction to any $C_{t}^{\prime}$ is of degree 0 . This determines a unique map

$$
\mu: T \longrightarrow J,
$$

and hence a map $\eta^{-1} \circ \mu: T \rightarrow \operatorname{Pic}_{C^{\prime} / k^{\prime}}^{d+g-1}$. Using the universal property of $J$ and $\mathcal{L}$ and the fact that the projection $p_{1}$ commutes with $\left(\operatorname{id}_{C^{\prime}} \times \eta\right)$, we see that the line bundle $\left(\operatorname{id}_{C^{\prime}} \times\left(\eta^{-1} \circ \mu\right)\right)^{*} \mathcal{P}$ is isomorphic to $\mathcal{L}_{T}$. Furthermore, the morphism $\eta^{-1} \circ \mu$ is unique with respect to this property. This is the universal property of $\mathcal{P}$.

Now let $\sigma$ be in $\operatorname{Gal}\left(k^{\prime} / k\right)$. If this induces an automorphism of a $k^{\prime}$-scheme, then it is also denoted by $\sigma$. For future purposes, we want to compute $\sigma^{*} \mathcal{P} \otimes \mathcal{P}^{-1}$. First we prove a computational lemma.

Lemma 2.11. With the notation as before, the following commutation relations hold:

(1) $\sigma \circ t_{(g-2)\left[P^{\sigma^{-1}}-P\right]} \circ m \circ\left(h \times(-1)_{J}\right) \circ(\mathrm{id} \times \eta)=m \circ\left(h \times(-1)_{J}\right) \circ(\mathrm{id} \times \eta) \circ \sigma$,

(2) $\sigma \circ t_{(-1)\left[P^{\sigma^{-1}}-P\right]} \circ p \circ\left(h \times(-1)_{J}\right) \circ(\mathrm{id} \times \eta)=p \circ\left(h \times(-1)_{J}\right) \circ(\mathrm{id} \times \eta) \circ \sigma$,

(3) $\sigma \circ t_{(g-1)\left[P^{\sigma^{-1}}-P\right]} \circ q \circ\left(h \times(-1)_{J}\right) \circ(\mathrm{id} \times \eta)=q \circ\left(h \times(-1)_{J}\right) \circ(\mathrm{id} \times \eta) \circ \sigma$. 
Proof. We prove only the first relation, since the others are proved in a similar way. Let $\mu_{l}$ (respectively $\mu_{r}$ ) denote the left (respectively the right) side of the first equation. Let $\mu=m \circ\left(\mu_{l},(-1) \circ \mu_{r}\right)$. Fix a closed point $Q$ on $C^{\prime}$ and a divisor $D$ associated to a line bundle that corresponds to a closed point in $\mathrm{Pic}_{C^{\prime} / k^{\prime}}^{d+g-1}$. Let $E$ denote the divisor of a section of $\mathcal{L}((g-1) P)(1)$. Then

$$
\begin{aligned}
\mu(Q, D) & \\
= & \mu_{l}(Q, D)-\mu_{r}(Q, D) \\
= & \sigma \circ t_{(g-2)\left[P^{-1}-P\right]} \circ m \circ\left(h \times(-1)_{J}\right)(Q, D-E) \\
& -m \circ\left(h \times(-1)_{J}\right) \circ(\mathrm{id} \times \eta)\left(Q^{\sigma}, D^{\sigma}\right) \\
= & \sigma \circ t_{(g-2)\left[P^{-1}-P\right]} \circ m([Q-P], E-D) \\
& -m \circ\left(h \times(-1)_{J}\right)\left(Q^{\sigma}, D^{\sigma}-E\right) \\
= & \left.\sigma \circ t_{(g-2)\left[P^{\sigma}-1\right.}-P\right] \\
= & {[Q-P]+E-D)-m \circ\left(Q^{\sigma}-P^{\sigma}+E^{\sigma}-D^{\sigma}+(g-2)\left(P-P^{\sigma}\right)\right]-\left[Q^{\sigma}-P+E-D^{\sigma}\right) }
\end{aligned}
$$

which is the empty divisor. (This follows from the observation that we may take $\left.E^{\sigma}-E=(g-1)\left[P^{\sigma}-P\right].\right)$ This means that $\mu(Q, D)$ is the trivial line bundle. So, by the rigidity lemma (14], Theorem 2.1), the two morphisms are the same.

Now let $\mathcal{P}$ be the universal bundle on $C^{\prime} \times{ }_{k^{\prime}} \operatorname{Pic}_{C^{\prime} / k^{\prime}}^{d+g-1}$, and let $\sigma$ be as above.

Lemma 2.12. Let $x$ be a closed point in $\operatorname{Pic}_{C^{\prime} / k^{\prime}}^{d+g-1}$ and $i: S p e c k(x) \rightarrow \operatorname{Pic}_{C^{\prime} / k^{\prime}}^{d+g-1}$ be the corresponding morphism. Then, with the above notation, we have that the line bundle $\left(\operatorname{id}_{C^{\prime}} \times i\right)^{*}\left(\sigma^{*} \mathcal{P} \otimes \mathcal{P}^{-1}\right)$ on $C^{\prime} \times k(x)$ is trivial. In other words, $\left(\sigma^{*} \mathcal{P} \otimes \mathcal{P}^{-1}\right)$ is isomorphic to $p_{2}^{*} L$, where $L$ is a line bundle on $\operatorname{Pic}_{C^{\prime} / k^{\prime}}^{d+g-1}$ and $p_{2}$ is the projection onto the second factor.

Proof. We first compute $\sigma^{*}\left(\operatorname{id}_{C^{\prime}} \times \eta\right)^{*} \mathcal{L} \otimes\left(\operatorname{id}_{C^{\prime}} \times \eta\right)^{*} \mathcal{L}^{-1}$. To this end, consider $\sigma^{*}\left(\operatorname{id}_{C^{\prime}} \times \eta\right)^{*} \mathcal{L}$. Using the definition of $\mathcal{L}$ and the relations of the last lemma, we have that

$$
\begin{aligned}
& \sigma^{*}\left(\operatorname{id}_{C^{\prime}} \times \eta\right)^{*} \mathcal{L} \\
&= \sigma^{*}\left(\operatorname{id}_{C^{\prime}} \times \eta\right)^{*}\left(h \times(-1)_{J}\right)^{*} \mathcal{L}^{\prime}\left(\Theta_{0}^{-}\right) \\
&= \sigma^{*}\left(\operatorname{id}_{C^{\prime}} \times \eta\right)^{*}\left(h \times(-1)_{J}\right)^{*}\left(m^{*} \mathcal{L}\left(\Theta_{0}^{-}\right) \otimes p^{*} \mathcal{L}\left(\Theta_{0}^{-}\right)^{-1} \otimes q^{*} \mathcal{L}\left(\Theta_{0}^{-}\right)^{-1}\right) \\
&=\left(\operatorname{id}_{C^{\prime}} \times \eta\right)^{*}\left(h \times(-1)_{J}\right)^{*}\left(m^{*} \mathcal{L}\left(\left(\Theta_{0}\right)_{-(g-2)\left[P^{\sigma^{-1}}-P\right]+(g-1)\left[P^{\sigma^{-1}}-P\right]}^{-}\right)\right. \\
& \otimes p^{*} \mathcal{L}\left(-\left(\Theta_{0}\right)_{\left[P^{\sigma^{-1}}-P\right]+(g-1)\left[P^{\sigma^{-1}}-P\right]}^{-}\right) \\
&\left.\otimes q^{*} \mathcal{L}\left(-\left(\Theta_{0}\right)_{-(g-1)\left[P^{\sigma^{-1}}-P\right]+(g-1)\left[P^{\sigma^{-1}}-P\right]}^{-}\right)\right) \\
&=\left(\operatorname{id}_{C^{\prime}} \times \eta\right)^{*}\left(h \times(-1)_{J}\right)^{*}\left(m^{*} \mathcal{L}\left(\left(\Theta_{0}\right)_{\left[P^{\sigma^{-1}}-P\right]}^{-}\right)\right. \\
& \otimes p^{*} \mathcal{L}\left(-\left(\Theta_{0}\right)_{g\left[P^{\sigma^{-1}}-P\right]}^{-}\right) \otimes q^{*} \mathcal{L}\left(-\left(\Theta_{0}\right)^{-}\right) .
\end{aligned}
$$

Now consider the line bundle $m^{*} \mathcal{L}\left(\left(\Theta_{0}\right)_{\left[P^{\sigma^{-1}}-P\right]}^{-}-\Theta_{0}^{-}\right)$. By [14], Proposition 10.1, the line bundle $\mathcal{L}\left(\left(\Theta_{0}\right)_{\left[P^{\sigma^{-1}}-P\right]}^{-}-\Theta_{0}^{-}\right)$lies in $\mathrm{Pic}^{0}\left(\mathrm{Pic}_{C^{\prime} / k^{\prime}}^{0}\right)$. But, by 14], Proposition 9.2, for any line bundle $\mathcal{L}^{\prime}$ on $\operatorname{Pic}_{C^{\prime} / k^{\prime}}^{0}$ that lies in $\operatorname{Pic}^{0}\left(\mathrm{Pic}_{C^{\prime} / k^{\prime}}^{0}\right)$, we have 
$m^{*} \mathcal{L}^{\prime} \cong p^{*} \mathcal{L}^{\prime} \otimes q^{*} \mathcal{L}^{\prime}$. Combining this fact with the above calculation, we have

$$
\begin{aligned}
& \sigma^{*}\left(\operatorname{id}_{C^{\prime}} \times \eta\right)^{*} \mathcal{L} \otimes\left(\operatorname{id}_{C^{\prime}} \times \eta\right)^{*} \mathcal{L}^{-1} \\
= & \left(\operatorname{id}_{C^{\prime}} \times \eta\right)^{*}\left(h \times(-1)_{J}\right)^{*} \\
& \left(p^{*} \mathcal{L}\left(\left(\Theta_{0}\right)_{\left[P^{\sigma^{-1}}-P\right]}^{-}-\left(\Theta_{0}\right)_{g\left[P^{\sigma^{-1}}-P\right]}^{-}\right) \otimes q^{*} \mathcal{L}\left(\left(\Theta_{0}\right)_{\left[P^{\sigma^{-1}}-P\right]}^{-}-\Theta_{0}^{-}\right)\right) .
\end{aligned}
$$

Recall that we are interested in the restriction of this line bundle to $C^{\prime} \times_{k^{\prime}} k(x)$. Since

$$
q \circ\left(h \times(-1)_{J}\right) \circ\left(\operatorname{id}_{C^{\prime}} \times \eta\right) \circ\left(\operatorname{id}_{C^{\prime}} \times i\right)=(-1)_{J} \circ \eta \circ i \circ q,
$$

it follows that the pull-back of the $q^{*}$-component of the above line bundle to $C^{\prime} \times_{k^{\prime}}$ $k(x)$ is trivial, and so we may consider only the $p^{*}$-component. Note that

$$
p \circ\left(h \times(-1)_{J}\right) \circ\left(\operatorname{id}_{C^{\prime}} \times \eta\right) \circ\left(\operatorname{id}_{C^{\prime}} \times i\right)=h \circ p_{1} .
$$

This gives us

$$
\begin{aligned}
& \left(\operatorname{id}_{C^{\prime}} \times i\right)^{*}\left(\sigma^{*}\left(\operatorname{id}_{C^{\prime}} \times \eta\right)^{*} \mathcal{L} \otimes\left(\operatorname{id}_{C^{\prime}} \times \eta\right)^{*} \mathcal{L}^{-1}\right) \\
\cong & p_{1}^{*} h^{*} \mathcal{L}\left(\left(\Theta_{0}\right)_{\left[P^{\sigma^{-1}}-P\right]}^{-}-\left(\Theta_{0}\right)_{g\left[P^{\sigma^{-1}}-P\right]}^{-}\right) .
\end{aligned}
$$

Now let $h^{(g)}$ denote the morphism $\left(C^{\prime}\right)^{(g)} \rightarrow \mathrm{Pic}_{C^{\prime} / k^{\prime}}^{0}$, which maps an effective divisor $D$ of degree $g$ to the line bundle associated to $[D-g P]$. Then it is clear that $h^{(g)}\left(P^{\sigma^{-1}}+(g-1) P\right)=\left[P^{\sigma^{-1}}-P\right]$ and $h^{(g)}\left(g P^{\sigma^{-1}}\right)=g\left[P^{\sigma^{-1}}-P\right]$. So by [15], Lemma 6.8, the invertible sheaf $h^{*} \mathcal{L}\left(\left(\Theta_{0}\right)_{\left[P^{\sigma^{-1}}-P\right]}^{-}\right)$is isomorphic to $\mathcal{L}\left(\left[P^{\sigma^{-1}}+(g-\right.\right.$ 1) $P])$, and $h^{*} \mathcal{L}\left(\left(\Theta_{0}\right)_{g\left[P^{\sigma^{-1}}-P\right]}^{-}\right) \cong \mathcal{L}\left(g\left[P^{\sigma^{-1}}\right]\right)$. Using [2.1] and these remarks, we get

$$
\begin{aligned}
& \left(\operatorname{id}_{C^{\prime}} \times i\right)^{*}\left(\sigma^{*}\left(\operatorname{id}_{C^{\prime}} \times \eta\right)^{*} \mathcal{L} \otimes\left(\operatorname{id}_{C^{\prime}} \times \eta\right)^{*} \mathcal{L}^{-1}\right) \\
\cong & p_{1}^{*} \mathcal{L}\left(P^{\sigma^{-1}}+(g-1) P-g P^{\sigma^{-1}}\right) .
\end{aligned}
$$

Now we consider the line bundle $\left(\operatorname{id}_{C^{\prime}} \times i\right)^{*}\left(\sigma^{*} \mathcal{P} \otimes \mathcal{P}^{-1}\right)$ on $C^{\prime} \times_{k^{\prime}} k(x)$. To finish the lemma, we consider the line bundle $p_{1}^{*}\left(L_{0}\right)^{-1}$. (Note that this $p_{1}$ is the projection of $C^{\prime} \times \operatorname{Pic}_{C^{\prime} / k^{\prime}}^{d+g-1}$ onto the first factor. We continue with this notation to avoid complicating our notation even further.) It follows from the definition of $L_{0}$ that

$$
\begin{aligned}
\left(\operatorname{id}_{C^{\prime}}\right. & \times i)^{*}\left(\sigma^{*} p_{1}^{*}\left(L_{0}\right)^{-1} \otimes p_{1}^{*}\left(L_{0}\right)\right) \\
& \cong\left(\operatorname{id}_{C^{\prime}} \times i\right)^{*} \mathcal{L}\left((g-1)\left(P^{\sigma^{-1}}-P\right)\right) \\
& \cong p_{1}^{*} \mathcal{L}\left((g-1)\left(P^{\sigma^{-1}}-P\right)\right) .
\end{aligned}
$$

Combining (2.2), 2.3 and the definition of $\mathcal{P}$, we see that the line bundle

$$
\left(\operatorname{id}_{C^{\prime}} \times i\right)^{*}\left(\sigma^{*} \mathcal{P} \otimes \mathcal{P}^{-1}\right)
$$

on $C^{\prime} \times k^{\prime} k(x)$ is trivial.

The last part of the statement now follows from the Seesaw Theorem, Cor. 6, Sect. 5, Chap. 2 in 18 . 
2.2. Auxiliary lemmas about rigidifications of line bundles. The following lemma will be used repeatedly in later sections.

Lemma 2.13. Let $C / k$ be a curve as before with a $k$-rational point, $\varepsilon: S p e c k \rightarrow C$. Let $K$ be a field extension of $k$. Then any two line bundles $\mathcal{L}_{1}, \mathcal{L}_{2}$ on $C_{K}$ that are rigidified along $\varepsilon_{K}$ are isomorphic as rigidified line bundles if they are isomorphic as line bundles.

Another lemma, which will be used later, concerns line bundles on $C \times_{k} D$, where $D=k[t] /\left(t^{2}\right)$ is the ring of dual numbers. However, for later purposes, it is sufficient to consider the case when $k$ is algebraically closed.

Fix a section $\varepsilon:$ Spec $k \rightarrow C$. We denote the induced section $\operatorname{Pic}_{C / k}^{d+g-1} \rightarrow$ $C \times{ }_{k} \operatorname{Pic}_{C / k}^{d+g-1}$ by $\varepsilon_{\mathrm{Pic}_{C / k}^{d+g-1}}$. Let $x$ be a closed point of $\mathrm{Pic}_{C / k}^{d+g-1}$, and let

$$
t_{x}: \operatorname{Spec} D \longrightarrow \operatorname{Pic}_{C / k}^{d+g-1}
$$

be a tangent vector at $x$. This gives a rigidified line bundle $\mathcal{L}_{1}$ on $C \times_{k} D$. Suppose that $\mathcal{L}_{2}$ is another line bundle on $C \times_{k} D$ such that we have an isomorphism

$$
\eta: \mathcal{L}_{1} \longrightarrow \mathcal{L}_{2}
$$

of line bundles. Further suppose that $\mathcal{L}_{2}$ is isomorphic (as a line bundle) to $j^{*} \mathcal{L}_{2}^{\prime}$ for some line bundle $\mathcal{L}_{2}^{\prime}$ on $C$, where $j$ is the morphism Spec $D \rightarrow$ Spec $k$ corresponding to the natural morphism $k \hookrightarrow D$.

Lemma 2.14. Under the above hypothesis and notation, there exists a rigidification on $\mathcal{L}_{2}^{\prime}$ such that $\mathcal{L}_{1}$ and $j^{*} \mathcal{L}_{2}^{\prime}$ are isomorphic as rigidified line bundles. Here $j^{*} \mathcal{L}_{2}^{\prime}$ is rigidified using the rigidification on $\mathcal{L}_{2}^{\prime}$ in a natural way.

Remark 2.15. We omit proofs of these lemmas. The first lemma can be proved by comparing the set of rigidifications of $\mathcal{L}_{2}$ with the automorphism group of $\mathcal{L}_{2}$. The second lemma can be proved by comparing the sets of rigidifications of $\mathcal{P}$, $j^{*} \mathcal{L}_{2}^{\prime}$ and $\mathcal{L}_{2}^{\prime}$ with automorphism groups of $\mathcal{O}_{\operatorname{Pic}_{C / k}^{d+g-1}}, \mathcal{O}_{D}$ and $\mathcal{O}_{k}$. However, the proofs are easier if we use yet another definition of the relative Picard functor. From 2, Section 8.1, the relative Picard functor is isomorphic to the functor which associates to an $S$-scheme $T$ the group $\operatorname{Pic}\left(X_{T}\right) / \operatorname{Pic}(T)$. In fact, this shows that two rigidifiable line bundles on $X_{T}$ that are isomorphic are isomorphic as rigidified line bundles.

\section{The complement of the $\Theta$-Divisor and the universal line Bundle}

In this section, we discuss the complement of the $\Theta$-divisor. However, after some generalities, we consider only those curves which are of interest from the point of view of the Clifford algebra. In particular, the projection of the restriction of the universal bundle to $C \times_{k}$ (complement of the $\Theta$-divisor) on $\mathbb{P}_{k}^{1} \times_{k}$ (complement of the $\Theta$-divisor) will be considered in detail. (The projection $C \rightarrow \mathbb{P}_{k}^{1}$ is described below.) We continue with the notation and definitions from the last section.

We first prove that the complement of the $\Theta$-divisor is an affine scheme. This is an immediate consequence of well-known properties of the $\Theta$-divisor. 
Proposition 3.1. Let $C / k$ be a degree $d$, genus $g$ smooth curve in $\mathbb{P}^{2}$. ( $C$ need not have a $k$-rational point.) Then the complement of the $\Theta$-divisor in $\operatorname{Pic}_{C / k}^{d+g-1}$ is an affine scheme.

Proof. First note that we may assume that $C$ has a $k$-rational point. This is because the $\Theta$-divisor and its complement were obtained by Galois descent on $\mathrm{Pic}_{C^{\prime} / k^{\prime}}^{d+g-1}$, where $k^{\prime} / k$ is an extension so that $C\left(k^{\prime}\right)$ is nonempty.

Suppose $C$ has a $k$-rational point. Then the $\Theta$-divisor is ample in $\operatorname{Pic}_{C / k}^{d+g-1}$. See [15], Remark 6.5 and Theorem 6.6. Now the proposition follows, since the complement of any ample divisor in $\mathrm{Pic}_{C / k}^{d+g-1}$ is an affine scheme.

Remark 3.2. If $C$ has a $k$-rational point, then the same proof shows that the complement of any $\Theta$-divisor in $\operatorname{Pic}_{C / k}^{m}$ is affine for any $m$.

Notation. For any curve $C / k$ as above, we denote by $S p e c A_{k}$ the open complement of the $\Theta$-divisor in $\operatorname{Pic}_{C / k}^{d+g-1}$.

For the rest of this section we assume that $C$ has a k-rational point.

Recall from the introduction that we are mainly interested in the curve $C=$ $\operatorname{Proj}\left(k[u, v, w] /\left(w^{d}-f(u, v)\right)\right.$. (Here $f$ is the given binary form.) We prove that in the cases of interest, the curve $C$ is always nonsingular.

Lemma 3.3. The curve $C$ is nonsingular provided that $f$ does not have repeated factors over an algebraic closure of $k$ and that the characteristic of $k$ does not divide $d$.

Proof. We may assume that $k$ is algebraically closed. An easy computation shows that

$$
\frac{\partial\left(w^{d}-f(u, v)\right)}{\partial u}=-\frac{\partial f}{\partial u}, \quad \frac{\partial\left(w^{d}-f(u, v)\right)}{\partial v}=-\frac{\partial f}{\partial v}
$$

and that $\frac{\partial\left(w^{d}-f(u, v)\right)}{\partial w}=d w^{d-1}$. Also we have that $u \frac{\partial g}{\partial u}+v \frac{\partial g}{\partial v}=d g$ for any polynomial $g(u, v)$. Since the characteristic of $k$ does not divide $d, C$ is singular only if the system of equations $\left\{f, \frac{\partial f}{\partial u}, \frac{\partial f}{\partial v}\right\}$ has a solution. However, since $f$ has no repeated factors, this is impossible.

Corresponding to the inclusion $k[u, v] \rightarrow\left(k[u, v, w] /\left(w^{d}-f(u, v)\right)\right.$ we have the projection

$$
C \stackrel{q_{k}}{\longrightarrow} \mathbb{P}_{k}^{1}
$$

For any field extension $K / k$, we denote the morphism obtained as above by $q_{K}$. Let $y$ be a closed point in $\operatorname{Spec} A_{k}$, and let $k(y)$ be its residue field. Then we denote by $\mathcal{L}_{y}$ the pull-back of the universal line bundle $\mathcal{P}$ under the canonical morphism

$$
C \times{ }_{k} k(y) \longrightarrow C \times{ }_{k} \mathrm{Pic}_{C / k}^{d+g-1}
$$

The following proposition is a key proposition for our purposes and was proved in [22] when $k$ is algebraically closed. We give a proof for the sake of completeness.

Proposition 3.4. The coherent sheaf $\mathcal{F}_{y}=\left(q_{k(y)}\right)_{*} \mathcal{L}_{y}$ is isomorphic to the trivial vector bundle $\bigoplus_{d} \mathcal{O}_{\mathbb{F}_{k(y)}^{1}}$. 
Proof. We first assume that $k$ is algebraically closed. Since $\mathbb{P}_{k}^{1}$ is a nonsingular curve over an algebraically closed field, any torsion-free coherent sheaf is a vector bundle. Since $\left(q_{k}\right)_{*}$ respects torsion-freeness ([6] I, Proposition 8.4.1), $\mathcal{F}_{y}$ is a vector bundle on $\mathbb{P}_{k}^{1}$. Since any vector bundle on $\mathbb{P}_{k}^{1}$ is a sum of line bundles, we can write $\mathcal{F}_{y} \cong \bigoplus_{i=1}^{d} \mathcal{O}_{\mathbb{P}_{k}^{1}}\left(n_{i}\right)$ for some integer $n_{i}$ 's. Note that $\chi\left(\mathcal{L}_{y}\right)=\chi\left(\mathcal{F}_{y}\right)$. Then, by [5], Cor. 15.2.1,

$$
\begin{aligned}
\chi\left(\mathcal{F}_{y}\right) & =\left(\operatorname{rank}\left(\mathcal{F}_{y}\right)\right)\left(1-g_{\mathbb{P}_{k}^{1}}\right)+\operatorname{deg}\left(\mathcal{F}_{y}\right), \\
\left(1-g_{C}\right)+\operatorname{deg}\left(\mathcal{L}_{y}\right) & =d+\sum_{i} n_{i} .
\end{aligned}
$$

Here $\operatorname{deg}(\mathcal{E})$ denotes the degree of a vector bundle $\mathcal{E}$. This gives $\sum_{i} n_{i}=0$. Since $y$ is in the complement of the $\Theta$-divisor, we have

$$
\begin{aligned}
h^{0}\left(\mathbb{P}_{k}^{1},\left(q_{k}\right)_{*}\left(\mathcal{L}_{y} \otimes_{\mathcal{O}_{C}} \mathcal{O}_{C}(-1)\right)\right) & =h^{0}\left(\mathbb{P}_{k}^{1},\left(q_{k}\right)_{*}\left(\mathcal{L}_{y}(-1)\right)\right) \\
& =h^{0}\left(C, \mathcal{L}_{y}(-1)\right),
\end{aligned}
$$

which is zero. But by the projection formula, we have the identity

$$
\left(q_{k}\right)_{*}\left(\mathcal{L}_{y} \otimes_{\mathcal{O}_{C}}\left(q_{k}^{*}\right) \mathcal{O}_{\mathbb{P}_{k}^{1}}(-1)\right) \cong\left(q_{k}\right)_{*} \mathcal{L}_{y} \otimes_{\mathcal{O}_{\mathbb{P}_{k}^{1}}} \mathcal{O}_{\mathbb{P}_{k}^{1}}(-1) .
$$

Using this and the previous equations gives

$$
h^{0}\left(\mathbb{P}_{k}^{1}, \bigoplus_{i} \mathcal{O}_{\mathbb{P}_{k}^{1}}\left(n_{i}-1\right)\right)=0
$$

so $n_{i} \leq 0$ for all $i=1, \cdots, d$, and hence $n_{i}=0$ (since $\left.\sum_{i} n_{i}=0\right)$.

So $\left(q_{k}\right)_{*} \mathcal{L}_{y} \cong \bigoplus_{d} \mathcal{O}_{\mathbb{P}_{k}^{1}}$. Now let $k$ be any field, and let $y$ be a point as before. Then we consider the (canonically rigidified) pull-back of the line bundle $\mathcal{L}_{y}$ under the canonical morphism $C \times_{k} \bar{k} \stackrel{j_{C}}{\rightarrow} C \times_{k} k(y)$. Then by Corollary 2.7, the image of the point corresponding to $j_{C}^{*} \mathcal{L}_{y}$ lies in $\operatorname{Spec} A_{\bar{k}}$. By the earlier part of the argument, $\left(q_{\bar{k}}\right)_{*}\left(j_{C}^{*}\left(\mathcal{L}_{y}\right)\right)=\bigoplus_{d} \mathcal{O}_{\mathbb{P}_{\bar{k}}^{1}}$. To finish the proof we consider the following commutative diagram:

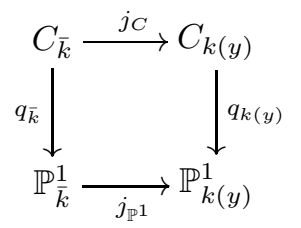

Now $\left(q_{\bar{k}}\right)_{*}\left(j_{C}^{*}\left(\mathcal{L}_{y}\right)\right) \cong\left(j_{\mathbb{P}^{1}}^{*}\right)\left(q_{k(y)}\right)_{*} \mathcal{L}_{y}$ by [11], Chapter III, Theorem 9.3. But $\operatorname{Aut}\left(\left(j_{\mathbb{P}^{1}}^{*}\right)\left(q_{k(y)}\right)_{*} \mathcal{L}_{y}\right)=\mathrm{GL}_{d}(\bar{k})$ and $H^{1}\left(\operatorname{Gal}_{\bar{k} / k(y)}, \mathrm{GL}_{d}(\bar{k})\right)=0$ by Hilbert 90 . So it follows that $\mathcal{F}_{y} \cong \bigoplus_{d} \mathcal{O}_{\mathbb{P}_{k(y)}^{1}}$.

Next we want to consider the direct image of the universal line bundle on $C \times_{k}$ $\operatorname{Pic}_{C / k}^{d+g-1}$ under the projection onto the second factor. In general, there is no reason for this to be a vector bundle. For our purposes, the restriction of this direct image to $S p e c A_{k}$ is more important. With the help of the last proposition we prove the following.

Proposition 3.5. Let $\pi: C \times{ }_{k} \operatorname{Pic}_{C / k}^{d+g-1} \rightarrow \mathrm{Pic}_{C / k}^{d+g-1}$ denote the projection onto the second factor. Then $\left.\left(\pi_{*} \mathcal{P}\right)\right|_{\text {Spec } A_{k}}$ is a locally free sheaf of rank $d$. 
Proof. First note that since Spec $A_{k} \rightarrow \operatorname{Pic}_{C / k}^{d+g-1}$ is an open immersion,

$$
\left.\pi_{*}\left(\left.\mathcal{P}\right|_{C \times_{k} \operatorname{Spec} A_{k}}\right) \cong\left(\pi_{*} \mathcal{P}\right)\right|_{\text {Spec } A_{k}} \text {. }
$$

For any point $y$ in Spec $A_{k}$, let $k(y)$ be the residue field of $y$. We denote by $\mathcal{P}_{y}$ the line bundle $(\mathrm{id} \times i)^{*} \mathcal{P}$ on $C_{k(y)}$, where $i$ is the morphism $\operatorname{Spec} k(y) \rightarrow \mathrm{Pic}_{C / k}^{d+g-1}$. We will prove that $\operatorname{dim}_{k(y)} H^{0}\left(C_{k(y)}, \mathcal{P}_{y}\right)$ is constant. Since $S p e c A_{k}$ is an open set in an integral scheme $\operatorname{Pic}_{C / k}^{d+g-1}$, it is itself irreducible and reduced. Then by [18], Corollary 2 , p. 50 , it follows that $\left.\left(\pi_{*} \mathcal{P}\right)\right|_{\text {Spec } A_{k}}$ is a locally free sheaf of rank $d$.

First we consider a closed point $y$ in Spec $A_{k}$. Then, with the notation from diagram (3.1),$\left(q_{k(y)}\right)_{*} \mathcal{P}_{y}$ is a trivial rank $d$ vector bundle. So

$$
h^{0}\left(C_{k(y)}, \mathcal{P}_{y}\right)=h^{0}\left(\mathbb{P}_{k(y)}^{1},\left(q_{k(y)}\right)_{*} \mathcal{P}_{y}\right)=d
$$

This holds for all closed points in Spec $A_{k}$. By the upper semicontinuity of the function $y \mapsto \operatorname{dim}_{k(y)} H^{0}\left(C_{k(y)}, \mathcal{P}_{y}\right)$ and the fact that Spec $A_{k}$ is a Jacobson scheme (in particular, any open set contains a closed point), it follows that $\operatorname{dim}_{k(y)} H^{0}\left(C_{k(y)}, \mathcal{P}_{y}\right)$ is constant.

The locally free sheaf on Spec $A_{k}$ in the statement of the previous proposition will be denoted by $\mathcal{E}$. As part of the proof of the last proposition we proved that the function $y \mapsto \operatorname{dim}_{k(y)} H^{0}\left(C_{k(y)}, \mathcal{P}_{y}\right)$ is constant. This, combined with [18], Corollary 2 , p. 50, gives the following corollary.

Corollary 3.6. For all $y \in S p e c A_{k}$, the natural map

$$
\mathcal{E} \otimes_{\mathcal{O}_{\text {Spec } A_{k}}} k(y) \longrightarrow H^{0}\left(C_{k(y)}, \mathcal{P}_{y}\right)
$$

is an isomorphism.

We have the following sequence of morphisms:

$$
C \times{ }_{k} \text { Spec } A_{k} \stackrel{q_{A_{k}}}{\longrightarrow} \mathbb{P}_{\times_{k}}^{1} \text { Spec } A_{k} \stackrel{p_{A_{k}}}{\longrightarrow} \text { Spec } A_{k} .
$$

We continue to denote by $\pi$ the projection onto the second factor of $C \times \operatorname{Spec} A_{k}$ (so that $p_{A_{k}} \circ q_{A_{k}}=\pi$ ). Let $\mathcal{F}$ denote the coherent sheaf $\left(q_{A_{k}}\right)_{*} \mathcal{P}$ on $\mathbb{P}_{k}^{1} \times{ }_{k}$ Spec $A_{k}$. The following lemma justifies this notation (compare Proposition 3.4).

Lemma 3.7. For any closed point $y$ in Spec $A_{k}$, let $i:$ Spec $k(y) \rightarrow$ Spec $A_{k}$ be the corresponding inclusion. Consider the Cartesian square

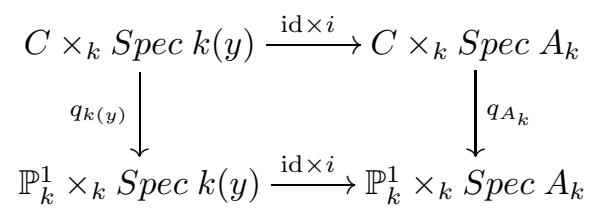

Then the coherent sheaves $(\mathrm{id} \times i)^{*}\left(q_{A_{k}}\right)_{*} \mathcal{P}$ and $\left(q_{k(y)}\right)_{*}(\mathrm{id} \times i)^{*} \mathcal{P}$ are isomorphic.

Proof. Since $\mathcal{P}$ is a coherent sheaf on the projective scheme $C \times_{k}$ Spec $A_{k}$ (over Spec $\left.A_{k}\right)$, there exists a graded $\left(A_{k}[u, v, w] /\left(w^{d}-f\right)\right)$-module $M$ such that $\tilde{M}$ is

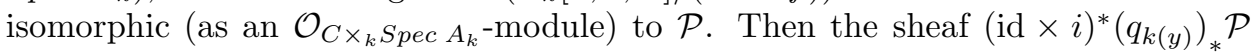
is isomorphic to $\left(k(y)[u, v]\left(k(y) \otimes A_{k} M\right)\right)^{\sim}$. Also the sheaf $\left(q_{k(y)}\right)_{*}(\mathrm{id} \times i)^{*} \mathcal{P}$ is isomorphic to the sheaf $\left(k(y) \otimes_{A_{k} A_{k}[u, v]} M\right)^{\sim}$. These conclusions follow from [6] II, Proposition 2.8.10. But the graded $k(y)[u, v]$-modules $\left(k(y)[u, v]\left(k(y) \otimes_{A_{k} A_{k}} M\right)\right)$ and $\left(k(y) \otimes_{A_{k} A_{k}[u, v]} M\right)$ are isomorphic. So the sheaves in question are isomorphic. 
Our goal for this section is to find a graded $A_{k}[u, v]$-module whose associated sheaf is isomorphic to $\mathcal{F}$. In fact, we will compute the module associated to $\mathcal{F}$, which will be sufficient. The next proposition is the main tool in this computation. Recall that for any morphism $g: X \rightarrow Y$ and a sheaf $\mathcal{G}$ of $\mathcal{O}_{X}$-modules, there is a natural morphism $g^{*} g_{*} \mathcal{G} \rightarrow \mathcal{G}$. This follows from the adjointness of the functors $g^{*}$ and $g_{*}$.

Proposition 3.8. With the notation as before, the natural morphism

$$
u: p_{A_{k}}^{*}\left(p_{A_{k}}\right)_{*} \mathcal{F} \longrightarrow \mathcal{F}
$$

is an isomorphism.

Proof. Recall that $\left(p_{A_{k}}\right)_{*} \mathcal{F}=\mathcal{E}$ is a locally free sheaf of rank $d$. So the sheaf $p_{A_{k}}^{*}\left(p_{A_{k}}\right)_{*} \mathcal{F}$ is also a locally free sheaf of rank $d$ on $\mathbb{P}_{A_{k}}^{1}\left(\mathbb{P}_{A_{k}}^{1}=\mathbb{P}_{k}^{1} \times_{k}\right.$ Spec $\left.A_{k}\right)$.

First we claim that $\mathcal{F}$ is a locally free sheaf of rank $d$ on $\mathbb{P}_{A_{k}}^{1}$. We prove that $\operatorname{dim}_{k(y)} \mathcal{F} \otimes_{\mathcal{O}_{\mathbb{P}_{A_{k}}}} k(y)$ is $d$ for any closed point $y$ in $\mathbb{P}_{A_{k}}^{1}$. Indeed by the upper semicontinuity of the dimension function and by the fact that $\mathbb{P}_{A_{k}}^{1}$ is of finite type over $k$, this will be sufficient.

Now for any closed point $y$ in $\mathbb{P}_{A_{k}}^{1}$, consider the following diagram:

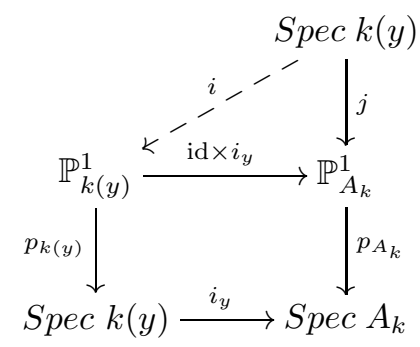

By the universal property of the fibre product, the dotted arrow $i$ exists so that this is a commutative diagram. We prove that

$$
\operatorname{dim}_{k(y)} \mathcal{F} \otimes_{\mathcal{O}_{\mathbb{P}^{1}}} k(y)=\operatorname{dim}_{k(y)} j^{*} \mathcal{F}
$$

is $d$. But

$$
\operatorname{dim}_{k(y)} j^{*} \mathcal{F}=\operatorname{dim}_{k(y)} i^{*}\left(\mathrm{id} \times i_{y}\right)^{*} \mathcal{F} .
$$

From Lemma 3.7 and Proposition 3.4 it follows that $\left(\mathrm{id} \times i_{y}\right)^{*} \mathcal{F}$ is a trivial vector bundle of rank $d$, so that $\operatorname{dim}_{k(y)} i^{*}\left(\operatorname{id} \times i_{y}\right)^{*} \mathcal{F}$ is $d$.

Now we go back to the morphism $u$ defined earlier. Notice that it is a morphism of vector bundles of rank $d$. To prove that $u$ is an isomorphism, it is sufficient to show that $u_{x}:\left(p_{A_{k}}^{*}\left(p_{A_{k}}\right)_{*} \mathcal{F}\right)_{x} \longrightarrow \mathcal{F}_{x}$ is bijective for all points $x$ in $\mathbb{P}_{A_{k}}^{1}$. By [6] I, Corollary 0.5.5.7, it is sufficient to prove that $u_{x}$ is surjective at closed points. The part about closed points follows from the fact that the set $\left\{x \in \mathbb{P}_{A_{k}}^{1} \mid u_{x}\right.$ is surjective $\}$ is open in $\mathbb{P}_{A_{k}}^{1}$. Then by [6] I, Corollary 0.5.5.6, it is sufficient to prove that

$$
u_{y} \otimes \mathrm{id}:\left(p_{A_{k}}^{*}\left(p_{A_{k}}\right)_{*} \mathcal{F}\right)_{y} / \mathfrak{m}_{y}\left(p_{A_{k}}^{*}\left(p_{A_{k}}\right)_{*} \mathcal{F}\right)_{y} \longrightarrow \mathcal{F}_{y} / \mathfrak{m}_{y} \mathcal{F}_{y}
$$

is surjective for all closed points $y$ in $\mathbb{P}_{A_{k}}^{1}$. But this homomorphism is surjective if and only if the morphism

$$
j^{*} u: j^{*} p_{A_{k}}^{*}\left(p_{A_{k}}\right)_{*} \mathcal{F} \longrightarrow j^{*} \mathcal{F}
$$


is surjective, which is the same as

$$
i^{*}\left(\mathrm{id} \times i_{y}\right)^{*} u: i^{*}\left(\mathrm{id} \times i_{y}\right)^{*} p_{A_{k}}^{*}\left(p_{A_{k}}\right)_{*} \mathcal{F} \longrightarrow i^{*}\left(\mathrm{id} \times i_{y}\right)^{*} \mathcal{F}
$$

being surjective. So it is sufficient to prove that

$$
\left(\mathrm{id} \times i_{y}\right)^{*} u:\left(\mathrm{id} \times i_{y}\right)^{*} p_{A_{k}}^{*}\left(p_{A_{k}}\right)_{*} \mathcal{F} \longrightarrow\left(\mathrm{id} \times i_{y}\right)^{*} \mathcal{F}
$$

is an isomorphism. But we have the isomorphism

$$
\left(\mathrm{id} \times i_{y}\right)^{*} p_{A_{k}}^{*}\left(p_{A_{k}}\right)_{*} \mathcal{F} \cong p_{k(y)}^{*} i_{y}^{*}\left(p_{A_{k}}\right)_{*} \mathcal{F} .
$$

Note that $i_{y}^{*}\left(p_{A_{k}}\right)_{*} \mathcal{F}$ is a trivial vector bundle of rank $d$, and so

$$
p_{k(y)}^{*} i_{y}^{*}\left(p_{A_{k}}\right)_{*} \mathcal{F} \cong p_{k(y)}^{*}\left(\bigoplus_{d} \mathcal{O}_{\text {Spec } k(y)}\right) \cong \bigoplus_{d} \mathcal{O}_{\mathbb{P}_{k(y)}^{1}}
$$

This gives that $\left(\mathrm{id} \times i_{y}\right)^{*} p_{A_{k}}^{*}\left(p_{A_{k}}\right)_{*} \mathcal{F}$ is a trivial vector bundle. Hence the morphism $\left(\mathrm{id} \times i_{y}\right)^{*} u$ will be an isomorphism if it is so on the global sections. But the morphism

$$
p_{k(y)}^{*} i_{y}^{*}\left(p_{A_{k}}\right)_{*} \mathcal{F} \longrightarrow\left(\mathrm{id} \times i_{y}\right)^{*} \mathcal{F}
$$

on the global sections is an isomorphism if the natural morphism

$$
\left(p_{A_{k}}\right)_{*} \mathcal{F} \otimes_{\mathcal{O}_{\text {Spec } A_{k}}} k(y) \longrightarrow H^{0}\left(\mathbb{P}_{k(y)}^{1}, \mathcal{F}_{y}\right)
$$

is an isomorphism. By the earlier part (dimension computation) of the proof and [18], Corollary 2, p. 50, this is indeed the case.

A useful application of this proposition is that it gives a convenient way to describe the graded module associated to the sheaf $\mathcal{F}$. Consider the sheaf $\left(p_{A_{k}}\right)_{*} \mathcal{F}=$ $\pi_{*}(\mathcal{P})$ on Spec $A_{k}$. As we saw before, this is a locally free sheaf of rank $d$ on Spec $A_{k}$. So we can find a projective $A_{k}$-module $P$ such that $\pi_{*}(\mathcal{P}) \cong \tilde{P}$.

Corollary 3.9. The $A_{k}[u, v]$-module $\bigoplus_{i} H^{0}\left(\mathbb{P}_{A_{k}}^{1}, \mathcal{F}(i)\right)$ is (graded) isomorphic to the module $M=P \otimes_{A_{k}} A_{k}[u, v]$.

Proof. It is sufficient to compute the graded module associated to the coherent sheaf $p_{A_{k}}^{*}\left(p_{A_{k}}\right)_{*} \mathcal{F}$, which we denote by $\mathcal{G}$. But, by the projection formula,

$$
\left(p_{A_{k}}\right)_{*}(\mathcal{G}(i)) \cong\left(p_{A_{k}}\right)_{*}(\mathcal{F}) \otimes\left(p_{A_{k}}\right)_{*}\left(\mathcal{O}_{\mathbb{P}_{A_{k}}^{1}}(i)\right)
$$

Since $\mathbb{P}_{A_{k}}^{1} \rightarrow$ Spec $A_{k}$ is a projective morphism, the module in the statement can be obtained by computing $H^{0}\left(\operatorname{Spec} A_{k},\left(p_{A_{k}}\right)_{*}(\mathcal{F}) \otimes\left(p_{A_{k}}\right)_{*}\left(\mathcal{O}_{\mathbb{P}_{A_{k}}^{1}}(i)\right)\right)$ for any $i$. From the above isomorphism it follows that

$$
\bigoplus_{i} H^{0}\left(\operatorname{Spec} A_{k},\left(p_{A_{k}}\right)_{*}(\mathcal{F}) \otimes\left(p_{A_{k}}\right)_{*}\left(\mathcal{O}_{\mathbb{P}_{A_{k}}^{1}}(i)\right) \cong P \otimes A_{k}\left(\bigoplus_{i}\left(p_{A_{k}}\right)_{*}\left(\mathcal{O}_{\mathbb{P}_{A_{k}}^{1}}(i)\right)\right)\right. \text {. }
$$

But now the statement follows from the well-known computation of the graded module associated to the structure sheaf of the projective $n$-space. See, for example, 11], Chapter V, Proposition 5.13. 


\section{The homomorphism $\tilde{C_{f}} \rightarrow \operatorname{End}_{A_{k}}(P)$}

We recall the set-up of the reduced Clifford algebra. Let $f$ be a binary form of degree $d$ over a field $k$ such that $\operatorname{char}(k)$ does not divide $d$. Then the Clifford algebra of the form $f$ was defined in the introduction. See Section 1 . One of the key properties of the representations of $C_{f}$ is that the dimension of the representation is divisible by $d$ ([10], Proposition 1.1). We are interested in representations of $C_{f}$ of dimension $d$. We form the reduced Clifford algebra $\tilde{C_{f}}$ by $\left(C_{f}\right) /(\cap \mathfrak{p})$, where the intersection is taken over the kernels of all dimension $d$ representations. The algebra $\tilde{C}_{f}$ is in fact an Azumaya algebra (for example, [10], Proposition 1.4), and so the $d$-dimensional representations are parametrized by the prime ideals in the center of $\tilde{C_{f}}$. Furthermore, it was pointed out to us that the center of $\tilde{C}_{f}$ is Noetherian. This, for example, follows from Proposition 2 of [17], since $C_{f}$ is finitely generated over $k$ and $\tilde{C}_{f}$ is a finite module over its center. We are trying to prove that the center of $\tilde{C_{f}}$ is isomorphic to the $k$-algebra $A_{k}$ of Section 3

In this section, we consider the case when the curve $C$ of Section 3 has a $k$ rational point. We construct a homomorphism from the center of $\tilde{C}_{f}$ to $A_{k}$. This is achieved by first constructing a homomorphism from $\tilde{C}_{f}$ to $\operatorname{End}_{A_{k}}(P)$, where $P$ is the module of the global sections of the sheaf $\pi_{*} \mathcal{P}$ on Spec $A_{k}$. See Section 3 We will show in the following sections that under this homomorphism the center of $\tilde{C_{f}}$ is mapped to $A_{k}$. After we have obtained the desired homomorphism, we investigate some of its properties. In particular, we want to show how, starting with a finite-dimensional representation of $\tilde{C}_{f}$, we can construct a line bundle over $C$ (possibly after a base extension).

Before we get to the main proposition of this section, we note an algebraic relation which holds inside the (graded) endomorphism ring of the graded module in Corollary[3.9. This relation will then enable us to define the morphism mentioned in the previous paragraph.

For the rest of this section, we assume that the curve $C$ has a k-rational point.

Lemma 4.1. Let $P$ be the projective $A_{k}$-module as in Corollary [3.9] Consider the graded $A_{k}[u, v]$-module $M=P \otimes_{A_{k}} A_{k}[u, v]$. Let $P_{i}$ denote the $i^{\text {th }}$ graded piece of this module (so $P \cong P_{0}$ ). Then we have the following equation:

$$
\operatorname{Hom}_{A_{k}}\left(P_{0}, P_{1}\right)=u \circ \operatorname{End}_{A_{k}} P_{0}+v \circ \operatorname{End}_{A_{k}} P_{0},
$$

where $u, v$ are viewed in $\operatorname{Hom}_{A_{k}}\left(P_{0}, P_{1}\right)$ with their natural action.

Proof. The proof is easy and follows from the relation $P_{1} \cong\left(P_{0} \oplus P_{0}\right)$, where the two generators are $u$ and $v$.

Proposition 4.2. There exists an algebra homomorphism

$$
\varphi: \tilde{C}_{f} \longrightarrow \operatorname{End}_{A_{k}} P
$$

where $\tilde{C_{f}}$ is the reduced Clifford algebra and $P$ is as defined before.

Proof. The strategy to prove this proposition will be to show that $\operatorname{End}_{A_{k}} P$ has elements that satisfy the relations of the Clifford algebra.

Consider the universal line bundle $\mathcal{P}$ on $C \times_{k} A_{k}$ and the projection $q_{A_{k}}: C \times_{k}$ $A_{k} \rightarrow \mathbb{P}_{A_{k}}^{1}$. Since $q_{A_{k}}$ is a projective (and hence an affine) morphism, we can obtain the graded module associated to $\mathcal{P}$ by computing $\bigoplus_{i} H^{0}\left(\mathbb{P}_{A_{k}}^{1},\left(q_{A_{k}}\right)_{*}(\mathcal{P}(i))\right)$. But this was already computed in Corollary [3.9. So the module $M$ of Lemma 4.1 is 
also a graded $A_{k}[u, v, w] /\left(w^{d}-f\right)$-module. Since $w$ is a homogeneous element of degree one in this (naturally) graded ring, it is an element of $\operatorname{Hom}_{A_{k}}\left(P_{0}, P_{1}\right)$. By Lemma 4.1, there exist elements $\alpha_{u}, \alpha_{v}$ in $\operatorname{End}_{A_{k}} P$ such that the equality

$$
w=u \circ \alpha_{u}+v \circ \alpha_{v}
$$

holds in $\operatorname{Hom}_{A_{k}}\left(P_{0}, P_{1}\right)$. Now consider the element $w^{d}$ as an element of the module $\operatorname{Hom}_{A_{k}}\left(P_{0}, P_{d}\right)$. Since in the graded ring of $C \times_{k} A_{k}$ we have the relation $w^{d}=f$, it holds in $\operatorname{Hom}_{A_{k}}\left(P_{0}, P_{d}\right)$ as well. In particular, we get

$$
w^{d}=\left(u \circ \alpha_{u}+v \circ \alpha_{v}\right)^{d}=f(u, v) \mathrm{Id} .
$$

But note that $\operatorname{Hom}_{A_{k}}\left(P_{0}, P_{d}\right)$ is a free $\operatorname{End}_{A_{k}} P_{0}$-module. This shows that the elements $\alpha_{u}$ and $\alpha_{v}$ in $\operatorname{End}_{A_{k}}\left(P_{0}\right)$ satisfy the relations of the Clifford algebra $C_{f}$ and give a homomorphism

$$
\tilde{\varphi}: C_{f} \longrightarrow \operatorname{End}_{A_{k}} P_{0}
$$

Next we want to show that this homomorphism factors through the reduced Clifford algebra. Recall that $S p e c A_{k}$ is an open subscheme in $\mathrm{Pic}_{C / k}^{d+g-1}$, which is integral. Thus $S p e c A_{k}$ is an integral scheme of finite type over $k$. In particular, $A_{k}$ is an integral domain. So we may consider the sequence of morphisms

$$
C_{f} \longrightarrow \operatorname{End}_{A_{k}}\left(P_{0}\right) \longrightarrow \operatorname{End}_{Q\left(A_{k}\right)}\left(P_{0} \otimes_{A_{k}} Q\left(A_{k}\right)\right) \cong M_{d}\left(Q\left(A_{k}\right)\right)
$$

where $Q\left(A_{k}\right)$ is the field of fractions of $A_{k}$. Note that, by the comments made above, the second arrow is an injection. This gives a $d$-dimensional representation of $C_{f}$. So the composite factors through $\tilde{C}_{f}$. We consider the diagram

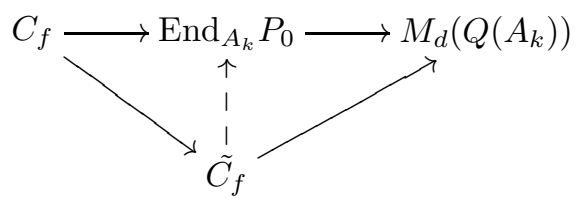

The dashed arrow exists since the second horizontal arrow is injective. This gives us the desired homomorphism

$$
\varphi: \tilde{C}_{f} \longrightarrow \operatorname{End}_{A_{k}} P_{0} .
$$

The homomorphism $\tilde{C}_{f} \rightarrow \operatorname{End}_{A_{k}} P_{0}$ constructed in the last proposition will always be denoted by $\varphi$.

Next we want to study the homomorphism $\varphi$. To this end, we assume that we are given a representation of $\tilde{C}_{f}$,

$$
\eta: \tilde{C}_{f} \longrightarrow M_{d}(K)
$$

where $K$ is a field extension of $k$. Our goal is to show that any such representation factors through $\operatorname{End}_{A_{k}} P_{0}$ via $\varphi$.

We denote the images of the two generators of $\tilde{C_{f}}$ under $\eta$ by $\overline{\alpha_{u}}$ and $\overline{\alpha_{v}}$. Consider the morphism

$$
\begin{aligned}
S_{K}=\frac{K[u, v, w]}{\left(w^{d}-f\right)} & \longrightarrow M_{d}(K[u, v]), \\
u, v & \longrightarrow[u],[v], \\
w & \longrightarrow \overline{\alpha_{u}}+v \overline{\alpha_{v}} .
\end{aligned}
$$


With the natural grading on $N=\bigoplus_{d} K[u, v]$, the above morphism is a graded homomorphism. This makes $N$ a graded $S_{K}$-module. So $\tilde{N}$ is a quasi-coherent (in fact, coherent) sheaf on $X=\operatorname{Proj} S_{K}$. But we can do better, as is seen in the next lemma. Note that $X$ is canonically isomorphic to $C_{K}=C \times_{k} K$.

Lemma 4.3. $\tilde{N}$ is an invertible sheaf on $X$. Moreover, the pull-back of this sheaf to $C_{K}$ under the canonical morphism is a degree $(d+g-1)$ line bundle on $C_{K}$. The image of the unique morphism Spec $K \rightarrow \mathrm{Pic}_{C / k}^{d+g-1}$ corresponding to this line bundle lies in Spec $A_{k}$.

Proof. For the first part of the statement, by [6], $4_{2}$, Proposition 2.5.1, it is sufficient to prove that $\tilde{N}$ is invertible with the assumption that $K$ is algebraically closed. We will prove that for any closed point $x \in X, \operatorname{dim}_{K}\left(\tilde{N} \otimes_{\mathcal{O}_{X, x}} K\right)=1$. This will be sufficient by the usual upper semicontinuity argument. Furthermore, it is clear that both $u$ and $v$ cannot be in a homogeneous maximal ideal of $S_{K}$. So it will be sufficient to prove the dimension condition for any closed point $x$ in $X_{v}=\operatorname{Spec}\left(\left(S_{K}\right)_{(v)}\right)$, since the argument for $X_{u}$ is similar. Now

$$
\left(S_{K}\right)_{(v)} \cong \frac{K[\bar{u}, \bar{w}]}{\left(\bar{w}^{d}-f(\bar{u}, 1)\right)} \text { and } N_{(v)} \cong \bigoplus_{d} K[\bar{u}]
$$

where $\bar{u}=u / v$ and $\bar{w}=w / v$. Here $\bar{u}$ acts in a natural way and $\bar{w}$ acts as $\bar{u} \overline{\alpha_{u}}+\overline{\alpha_{v}}$. Any closed point in $\operatorname{Spec}\left(\left(S_{K}\right)_{(v)}\right)$ is $\mathfrak{m}=(\bar{u}-a, \bar{w}-b)$ for some $a, b \in K$. So we have that

$$
\mathcal{O}_{\operatorname{Spec}\left(\left(S_{K}\right)_{(v)}\right), x} \cong\left(\frac{K[\bar{u}, \bar{w}]}{\left(\bar{w}^{d}-f(\bar{u}, 1)\right)}\right)_{(\bar{u}-a, \bar{w}-b)}
$$

as well as

$$
\left(N_{(v)}\right)_{x} \cong \mathcal{O}_{x} \otimes_{\left(S_{K}\right)_{(v)}}\left(\bigoplus_{d} K[\bar{u}]\right)
$$

This gives

$$
\begin{aligned}
(\tilde{N})_{x} \otimes_{\mathcal{O}_{X, x}} K & \cong \frac{\bigoplus_{d} K[\bar{u}]}{(\bar{u}-a, \bar{w}-b)\left(\oplus_{d} K[\bar{u}]\right)} \\
& \cong \frac{\bigoplus_{d} K}{\left(a \overline{\alpha_{u}}+\overline{\alpha_{v}}-b\right)\left(\oplus_{d} K\right)} .
\end{aligned}
$$

So the required dimension is $\operatorname{dim}_{K}\left(\operatorname{ker}\left(a \overline{\alpha_{u}}+\overline{\alpha_{v}}-b\right)\right)$. We consider $a \overline{\alpha_{u}}+\overline{\alpha_{v}} \in$ $M_{d}(K)$ and compute the dimension of its eigenspace of eigenvalue $b$. The characteristic polynomial of $a \overline{\alpha_{u}}+\overline{\alpha_{v}}$ is $t^{d}-f(a, 1)$. Indeed, this follows when $f(a, 1) \neq 0$ since all the roots are distinct ( $\operatorname{since} \operatorname{char}(K)$ does not divide $d$ ), and when $f(a, 1)=0$ since the matrix is nilpotent in this case. Moreover, if $b \neq 0$, then $f(a, 1) \neq 0$ and $b$ is an eigenvalue of multiplicity 1.

Let $b=0$, so that $f(a, 1)=0$. We may find a matrix $B \in \mathrm{GL}_{d}(K)$ such that $B\left(a \overline{\alpha_{u}}+\overline{\alpha_{v}}\right) B^{-1}$ is in its Jordan form. If $\operatorname{dim}_{K}\left(\operatorname{ker}\left(a \overline{\alpha_{u}}+\overline{\alpha_{v}}-b\right)\right)>1$, then we 
can write $\left(\right.$ for $\left.w \in M_{d}(K[u, v])\right) \operatorname{det} w=\operatorname{det} B w B^{-1}=(a v-u)^{l} \operatorname{det} w^{\prime}$, where

$$
B w B^{-1}=\left(\begin{array}{cccccc}
a v-u & \ldots & 0 & 0 & \ldots & 0 \\
\vdots & \ddots & \vdots & \vdots & \ddots & \vdots \\
0 & \ldots & a v-u & 0 & \ldots & 0 \\
0 & \ldots & 0 & 1 & \ldots & 0 \\
\vdots & \ddots & \vdots & \vdots & \ddots & \vdots \\
0 & \ldots & 0 & 0 & \ldots & 1
\end{array}\right) w^{\prime} .
$$

There are $l$ entries $(a v-u)$ in the above matrix, and $l \geq 2$. But this gives that $\operatorname{det} w^{d}=(a v-u)^{l d} \operatorname{det}\left(w^{\prime}\right)^{d}=f(u, v)^{d}$. Since $l \geq 2, a v-u$ is a repeated factor of $f(u, v)$, which contradicts our assumption on $f$. This gives the required dimension condition, and $\tilde{N}$ is an invertible sheaf on $C_{K}$.

For the second part, consider the projection $q_{K}: C_{K} \rightarrow \mathbb{P}_{K}^{1}$ considered before. Then

$$
\chi\left(C_{K}, \tilde{N}\right)=\chi\left(\mathbb{P}_{K}^{1},\left(q_{K}\right)_{*} \tilde{N}\right),
$$

and, by the Riemann-Roch formula for vector bundles on nonsingular curves ([5], Example 15.2.1),

$$
(1-g)+\operatorname{deg} \tilde{N}=d(1-0)+\operatorname{deg}\left(\left(q_{K}\right)_{*} \tilde{N}\right) .
$$

$\operatorname{But}\left(q_{K}\right)_{*} \tilde{N} \cong \bigoplus_{d} \mathcal{O}_{\mathbb{P}_{K}^{1}}$ and so $\operatorname{deg}\left(\left(q_{K}\right)_{*} \tilde{N}\right)=0$. This gives that $\operatorname{deg} \tilde{N}=$ $(d+g-1)$.

For the third part, note that by the projection formula,

$$
h^{0}\left(C_{K}, \tilde{N}(-1)\right)=h^{0}\left(\mathbb{P}_{K}^{1},\left(\left(q_{K}\right)_{*} \tilde{N}\right)(-1)\right)=0 .
$$

By Proposition 2.6, the image of Spec $K$ under the unique morphism corresponding to $\tilde{N}$ lies in $\operatorname{Spec} A_{k}$.

Remark 4.4. The above lemma is essentially the key lemma of [22]. As we will see later, it provides an explicit construction of line bundles on $C \times_{k} K$ arising from representations of $C_{f}$. It is the main tool in proving the one-to-one correspondence between representations of $\tilde{C}_{f}$ and line bundles on $C$ mentioned in the introduction (Section 1).

Now we get to the main proposition mentioned at the beginning of the section.

Proposition 4.5. Let $\eta: \tilde{C}_{f} \rightarrow M_{d}(K)$ be a representation. Then there exists a morphism $\operatorname{End}_{A_{k}} P_{0} \rightarrow M_{d}(K)$ so that the following diagram is commutative:

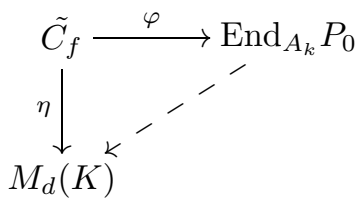

Proof. Given a finite-dimensional representation of $\tilde{C_{f}}$, we can construct a graded $S_{K}$-module $N$ as before, where $S_{K}=\left(K[u, v, w] /\left(w^{d}-f\right)\right)$. This gives a line bundle $\mathcal{L}$ on $C_{K}$ as in the last lemma. Then the image of the morphism corresponding to 
this line bundle lies in Spec $A_{k}$. Note that by Lemma 2.13, this morphism does not depend on the choice of rigidification. If we denote by $i$ the morphism

$$
\text { Spec } K \stackrel{i}{\longrightarrow} \operatorname{Spec} A_{k},
$$

then $(\text { id } \times i)^{*} \mathcal{P} \cong \mathcal{L}$, where $\mathcal{P}$ is the universal line bundle on $C \times_{k}$ Spec $A_{k}$. Recall that $\mathcal{P}$ is the sheaf associated to the graded module $\bigoplus_{i} P_{i}$, where $P_{i}=$ $H^{0}\left(C \times_{k}\right.$ Spec $\left.A_{k}, \mathcal{P}(i)\right)$. In fact,

$$
\bigoplus_{i} P_{i}=P \otimes_{A_{k}} A_{k}[u, v]
$$

So the line bundle $(\text { id } \times i)^{*} \mathcal{P} \cong \mathcal{L} \cong \tilde{N}^{\prime}$, where $N^{\prime}$ is the graded $S_{K}$-module $K \otimes_{A_{k}}\left(\bigoplus_{i} P_{i}\right)$. We claim that the $S_{K}$-modules $N^{\prime}$ and $N$ are isomorphic as graded modules.

For any line bundle $\mathcal{L}^{\prime}$, we denote by $\Gamma_{*}\left(\mathcal{L}^{\prime}\right)$ the associated graded module $\bigoplus_{i} H^{0}\left(\mathcal{L}^{\prime}(i)\right)$. We have the following diagram, in which the horizontal arrow exists by functoriality of $\Gamma_{*}$ and the vertical morphisms are natural:

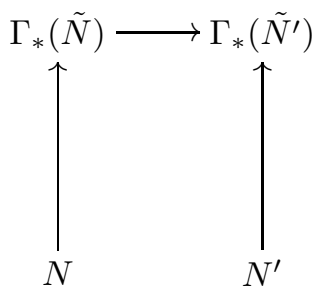

Since the sheaves associated to the graded modules $N, N^{\prime}$ are isomorphic, the horizontal arrow is an isomorphism (as $\Gamma_{*}\left(\mathcal{O}_{C_{K}}\right)$ and hence as $S_{K}$-modules). Thus it will be sufficient to prove that

(1) $N \cong \Gamma_{*}(\tilde{N})$ and

(2) $N^{\prime} \cong \Gamma_{*}\left(\tilde{N}^{\prime}\right)$

as $S_{K}$-modules. Note that the vertical morphisms are $S_{K}$-module maps, and hence it is sufficient to prove that these are bijective. Consider the first morphism as a $K[u, v]$-module morphism. By [11, Proposition 5.13 and the fact that $\Gamma_{*}$ commutes with formation of direct sum, it follows that the first morphism is an isomorphism. But a similar argument along with the observation that

$$
\begin{aligned}
N^{\prime} & \cong\left(K \otimes_{A_{k}} P_{0}\right) \otimes_{A_{k}} A_{k}[u, v] \\
& \cong\left(\bigoplus_{d} K\right) \otimes_{A_{k}} A_{k}[u, v] \\
& \cong \bigoplus_{d} K[u, v]
\end{aligned}
$$

shows that $N^{\prime} \rightarrow \Gamma_{*}\left(\tilde{N}^{\prime}\right)$ is bijective.

This gives a degree-preserving $S_{K}$-module isomorphism

$$
\bigoplus_{d} K[u, v]=N \stackrel{\mu}{\longrightarrow} N^{\prime}=\left(K \otimes_{A_{k}} P_{0}\right) \otimes_{A_{k}} A_{k}[u, v] .
$$

In particular, we get a $K$-module isomorphism

$$
\bigoplus_{d} K \stackrel{\bar{\mu}}{\longrightarrow} K \otimes_{A_{k}} P_{0},
$$


and hence an isomorphism

$$
M_{d}(K) \stackrel{\bar{\mu}-\bar{\mu}^{-1}}{\longrightarrow} \operatorname{End}_{K}\left(K \otimes_{A_{k}} P_{0}\right) .
$$

Since $\mu$ preserves the action of $w$, the image of $\overline{\alpha_{u}}$ (respectively $\overline{\alpha_{v}}$ ) is (id $\otimes \alpha_{u}$ ) (respectively $\left(\mathrm{id} \otimes \alpha_{v}\right)$ ). So we have a commutative diagram

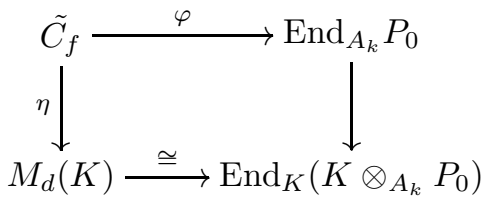

This is the desired diagram of the statement.

Remark 4.6. Note that the morphism $\operatorname{End}_{A_{k}} P_{0} \rightarrow M_{d}(K)$ obtained in the previous proposition by our procedure is uniquely determined up to a unique automorphism of $M_{d}(K)$. In fact, the only place where we do not have an explicit construction is in the use of the morphism Spec $K \rightarrow$ Spec $A_{k}$. However, this morphism is uniquely determined once we have constructed a line bundle $\tilde{N}$ as in the proof. This follows by Lemma 2.13. The rest of the procedure is algorithmic, and determines a unique homomorphism up to an element of $\mathrm{GL}_{d}(K)$.

We now use the above proposition to prove a very useful property of the morphism $\varphi$.

Corollary 4.7. The morphism

$$
\varphi: \tilde{C}_{f} \longrightarrow \operatorname{End}_{A_{k}} P_{0}
$$

constructed earlier is injective.

Proof. Let $I$ denote the kernel of the morphism $\varphi$. Then since any representation has a factorization as in Proposition 4.5, I must be contained in the intersection of the kernels of $d$-dimensional representations. Thus, since $\tilde{C}_{f}$ is an Azumaya algebra, $I$ is contained in the (two-sided) ideal generated by the nilradical of $Z_{k}$, the center of $\tilde{C}_{f}$. But the center $Z_{k}$ of $\tilde{C}_{f}$ is reduced; see [10], Proposition 1.4. So the ideal $I=(0)$, and $\varphi$ is injective.

4.1. The morphism $\varphi:$ Spec $A_{k} \longrightarrow \operatorname{Spec} Z_{k}$. Consider the morphism $\varphi: \tilde{C_{f}} \rightarrow$ $\operatorname{End}_{A_{k}} P_{0}$ as above.

Proposition 4.8. The morphism $\varphi: \tilde{C}_{f} \longrightarrow \operatorname{End}_{A_{k}} P_{0}$ maps the center $Z_{k}$ to $A_{k}$.

Proof. Consider the sequence of morphisms

$$
\tilde{C_{f}} \stackrel{\varphi}{\longrightarrow} \operatorname{End}_{A_{k}} P_{0} \stackrel{i}{\hookrightarrow} M_{d}\left(Q\left(A_{k}\right)\right) .
$$

Let $z \in Z_{k}$. We claim that $\varphi(z)$ is integral over $A_{k}$ and that $i \circ \varphi(z) \in Q\left(A_{k}\right)$.

The first claim follows since $\varphi(z)$ commutes with $A_{k}$ and $\operatorname{End}_{A_{k}} P_{0}$ is a finitely generated module over $A_{k}$. For the second claim, note that the composite $i \circ \varphi$ is an irreducible representation of $\tilde{C}_{f}$. Hence it follows that $(i \circ \varphi)\left(\tilde{C}_{f}\right) Q\left(A_{k}\right)=$ $M_{d}\left(Q\left(A_{k}\right)\right)$. Now the claim follows since this implies that $i \circ \varphi\left(Z_{k}\right) \subseteq Q\left(A_{k}\right)$.

But since Spec $A_{k}$ is a smooth integral scheme, $A_{k}$ is integrally closed, which implies that $\varphi(z) \in A_{k}$. 
The above proposition along with Corollary 4.7 gives an injective $k$-algebra morphism

$$
\varphi: Z_{k} \longrightarrow A_{k}
$$

and hence a $k$-morphism

$$
\varphi: \operatorname{Spec} A_{k} \longrightarrow \operatorname{Spec} Z_{k} .
$$

If there is no cause for confusion, we will denote both of the above morphisms by $\varphi$.

We will prove in the following sections that $\varphi$ is an isomorphism.

\section{The MorPhism $\eta: S p e c A_{k} \rightarrow S p e c Z_{k}$}

In this section, we construct a morphism $\eta:$ Spec $Z_{k} \rightarrow$ Spec $A_{k}$ which we will show to be the inverse of the morphism $\varphi$ constructed earlier. The main idea in constructing the morphism $\eta$ is to view the schemes $\operatorname{Spec} A_{k}$ and $\operatorname{Spec} Z_{k}$ as representing objects of certain functors, and then construct a natural transformation between these functors. These ideas are present in the paper [23] of M. Van den Bergh.

If $S$ is a $k$-scheme, then by an $S$-representation of degree $n$ of $\tilde{C_{f}}$ we mean a pair $\left(\psi, \mathcal{O}_{A}\right)$, where $\mathcal{O}_{A}$ is a sheaf of Azumaya algebras of rank $n^{2}$ over $S$ and $\psi$ : $\tilde{C_{f}} \rightarrow H^{0}\left(S, \mathcal{O}_{A}\right)$ is a $k$-algebra homomorphism. Two $S$-representations $\left(\psi_{1}, \mathcal{O}_{A_{1}}\right)$ and $\left(\psi_{2}, \mathcal{O}_{A_{2}}\right)$ are said to be equivalent if there is an isomorphism $\theta: \mathcal{O}_{A_{1}} \rightarrow \mathcal{O}_{A_{2}}$ of sheaves of Azumaya algebras such that $\psi_{2}=H^{0}(S, \theta) \circ \psi_{1}$. An $S$-representation of $\tilde{C}_{f}$ is irreducible if the image of $\tilde{C}_{f}$ generates $\mathcal{O}_{A}$ locally.

Let $\mathcal{R} e p_{d}\left(\tilde{C_{f}},-\right)$ be the functor that assigns to a $k$-scheme $S$ the set of equivalence classes of irreducible $S$-representations of degree $d$ of $\tilde{C}_{f}$. Since Azumaya algebras pull back to Azumaya algebras and irreducible representations are stable under pull-back, it follows that this is a functor. Furthermore, since $\tilde{C}_{f}$ is an Azumaya algebra of rank $d^{2}$ over its center, this functor is representable in Sch/k and is represented by Spec $Z_{k}$. Note that, by remarks at the beginning of Section 4 Spec $Z_{k}$ is a Noetherian scheme.

We also consider representations of $\tilde{C}_{f}$ into endomorphism sheaves of vector bundles. Let $\mathcal{G}_{d}\left(\tilde{C}_{f},-\right)$ be the subfunctor of $\mathcal{R} e p_{d}\left(\tilde{C}_{f},-\right)$ that assigns to a $k$-scheme $S$ the set of equivalence classes of irreducible $S$-representations into endomorphism sheaves of vector bundles of rank $d$. Again, since endomorphism sheaves of vector bundles pull back to the sheaves of the same kind, it follows that this is also a functor. Both the functors $\mathcal{R e p}_{d}\left(\tilde{C_{f}},-\right)$ and $\mathcal{G}_{d}\left(\tilde{C_{f}},-\right)$ define presheaves on $(\mathrm{Sch} / k)_{\mathrm{fl}}$ with respect to the flat topology. Moreover, the functor $\mathcal{R}_{e} p_{d}\left(\tilde{C}_{f},-\right)$ is a sheaf with respect to the flat topology, since it is representable (Proposition 1, Chapter 8, 2] $)$. We denote the sheafification of the functor $\mathcal{G}_{d}\left(\tilde{C}_{f},-\right)$ with respect to the flat topology by $\overline{\mathcal{G}}_{d}\left(\tilde{C_{f}},-\right)$. The following fact is [23, Lemma 4.2 .

Lemma 5.1. The natural transformation $\mathcal{G}_{d}\left(\tilde{C}_{f},-\right) \rightarrow \mathcal{R} e p_{d}\left(\tilde{C}_{f},-\right)$ induces an isomorphism $\mathcal{R e p}_{d}\left(\tilde{C}_{f},-\right) \cong \overline{\mathcal{G}}_{d}\left(\tilde{C}_{f},-\right)$.

Our next goal is to define a natural transformation from $\mathcal{R} e p_{d}\left(\tilde{C}_{f},-\right)$ to a certain open subfunctor of $\operatorname{Pic}_{C / k}^{d+g-1}(-)$. Before we define this morphism of functors, we need some auxiliary lemmas. 
Let $S$ be a $k$-scheme, and let $\left(\psi, \mathcal{O}_{A}\right)$ be an element of $\mathcal{G}_{d}\left(\tilde{C}_{f}, S\right)$ so that $\mathcal{O}_{A}=$ $\mathcal{E} n d_{\mathcal{O}_{S}}(\mathcal{E})$ for some vector bundle $\mathcal{E}$ of rank $d$ on $C \times_{k} S$. We will construct an invertible sheaf on $C \times_{k} S$. Consider the graded sheaf homomorphism

$$
\begin{aligned}
\frac{\mathcal{O}_{S}[u, v, w]}{w^{d}-f(u, v)} & \longrightarrow \mathcal{E} n d(\mathcal{E})[u, v], \\
u, v & \longrightarrow u, v, \\
w & \longrightarrow u \psi\left(x_{1}\right)+v \psi\left(x_{2}\right),
\end{aligned}
$$

where $x_{1}$ and $x_{2}$ are the usual generators of $\tilde{C}_{f}$. Since $\mathcal{E}$ is a sheaf of $\mathcal{O}_{S}$-modules, there is a canonical map from $\mathcal{O}_{S}$ to $\mathcal{E} n d(\mathcal{E})$. Since this is a graded homomorphism of graded algebras (degree of $u, v$ is 1 on the right side), we get a graded module $\mathcal{E} \otimes \mathcal{O}_{S} \mathcal{O}_{S}[u, v]$. This defines a sheaf $\mathcal{M}$ on $C \times_{k} S$. In the lemma below and in the remainder of this section, for any point $s$ in $S, q_{s}$ denotes the morphism $C \times{ }_{k} k(s) \rightarrow$ $\mathbb{P}_{k(s)}^{1}$ induced by the inclusion $k(s)[u, v] \rightarrow(k(s)[u, v, w]) /\left(w^{d}-f(u, v)\right), p_{s}$ denotes the projection of the second factor of $\mathbb{P}_{k(s)}^{1}$ onto Spec $k(s)$, and $\pi_{s}$ denotes the composition $p_{s} \circ q_{s}$. Similar notation is used for an arbitrary field $K$ instead of $k(s)$.

Lemma 5.2. The sheaf $\mathcal{M}$ is an invertible sheaf on $C \times_{k} S$ of (fibre-wise constant) degree $(d+g-1)$. Furthermore, the invertible sheaf $\mathcal{M}_{s}=\mathcal{M} \otimes_{\mathcal{O}_{S}}$ Spec $k(s)$ on $C_{k(s)}$ satisfies $h^{0}\left(C_{k(s)}, \mathcal{M}_{s}(-1)\right)=0$.

Proof. It is sufficient to prove the first assertion in the case when $S$ is an affine scheme. So let $S=\operatorname{Spec} R$. Let $C_{R}$ denote the scheme $C \times_{k} S$. We may assume that $H^{0}(S, \mathcal{E}) \cong \bigoplus_{d} R$, since $\mathcal{E}$ is a vector bundle of rank $d$. In this case, we denote the graded $\left(R[u, v, w] /\left(w^{d}-f(u, v)\right)\right.$-module $H^{0}(S, \mathcal{E}) \otimes_{R} R[u, v]$ by $M$ and the associated sheaf $\mathcal{M}$ by $\tilde{M}$.

Now consider the first assertion. Note that $\tilde{M}$ is flat over $S$ and $\pi$ is a flat morphism. So by [6], $4_{2}$, Lemma 12.3 .1 , it is sufficient to prove that for any point $s$ in $S, \tilde{M}_{s}$ is an invertible sheaf on $S$. Here $\tilde{M}_{s}$ is the sheaf $\left(1 \times i_{s}\right)^{*} \tilde{M}$ where $1 \times i_{s}$ is the morphism $C_{s}=C \times_{k} \operatorname{Spec} k(s) \rightarrow C \times_{k} S$. Consider the representation associated to the point $s$ via $\psi$ :

$$
\psi_{s}: \tilde{C}_{f} \longrightarrow M_{d}(k(s)) .
$$

This gives a sheaf $\tilde{N}$ as in the discussion preceding Lemma 4.3. The sheaf $\tilde{N}$ is isomorphic to the sheaf $\tilde{M}_{s}$. So the fact that $\tilde{M}_{s}$ is invertible now follows from Lemma 4.3. Also, the degree is $(d+g-1)$ along fibres of the projection onto the second factor. This follows from a calculation using the Riemann-Roch formula; see the proof of Lemma 4.3

For the second assertion, it is sufficient to prove the statement for the invertible sheaf $\tilde{N}$ of the last paragraph. But, in this case, $\left(q_{k(s)}\right)_{*} \mathcal{M}_{s}$ is a trivial vector bundle of rank $d$. This, along with the projection formula, gives the required statement. See Lemma 4.3 for details.

Corollary 5.3. With the notation as in Lemma 5.2, the resulting morphism $S \rightarrow$ $\mathrm{Pic}_{C / k}^{d+g-1}$ factors through Spec $A_{k}$.

Proof. Since the sheaf $\mathcal{M}$ is invertible on $C \times{ }_{k} S$, we have a morphism $S \rightarrow \mathrm{Pic}_{C / k}^{d+g-1}$ (after rigidifying this invertible sheaf if necessary). From Remark [2.15, this morphism does not depend on the rigidification. Now it is sufficient to prove that the 
image of any point $s$ in $S$ is in Spec $A_{k}$ under the morphism in the statement. However, it follows from Lemma 4.3 that the image of any point $s$ in $S$ lies in Spec $A_{k}$. So we get the required factorization.

Now we consider another functor $\mathcal{H}_{C / k}^{d+g-1}$ defined as follows. This will be defined as a subfunctor of $\operatorname{Pic}_{C / k}^{d+g-1}$. For any $k$-scheme $S$, let $\mathcal{H}_{C / k}^{d+g-1}(S)$ be the subset of $\operatorname{Pic}_{C / k}^{d+g-1}(S)$ associated to the set of rigidified line bundles $\mathcal{L}$ on $C \times_{k} S$ that satisfy the condition that for any point $s$ of $S, h^{0}\left(C_{s}, \mathcal{L}_{s}(-1)\right)=0$. Here $C_{s}=C \times_{k} k(s)$ and $\mathcal{L}_{s}$ denotes the sheaf $\left(1_{C} \times i(s)\right)^{*} \mathcal{L}$, where $i(s)$ is the inclusion $\operatorname{Spec} k(s) \rightarrow S$.

To see that this is indeed a functor, consider a $k$-scheme $T$ and a $k$-morphism $f: T \rightarrow S$, and let $\mathcal{L}$ be an invertible sheaf on $C_{S}=C \times_{k} S$ which gives an element of $\mathcal{H}_{C / k}^{d+g-1}(S)$. We need to prove that for any point $t$ in $T, h^{0}\left(C_{t}, \mathcal{L}_{t}(-1)\right)=0$. But if $s$ is the image of $t$, then we have $h^{0}\left(C_{s}, \mathcal{L}_{s}(-1)\right)=0$. Now the required dimension is zero, since the composition $f \circ i(t)$ factors through the inclusion $i(s)$ : $\operatorname{Spec} k(s) \rightarrow S$. It follows that this definition gives a functor. For this functor, we have the following lemma.

Lemma 5.4. The functor $\mathcal{H}_{C / k}^{d+g-1}$ is an open subfunctor of the functor $\mathrm{Pic}_{C / k}^{d+g-1}$. Furthermore, $\mathcal{H}_{C / k}^{d+g-1}$ is a representable functor and is represented by Spec $A_{k}$.

Proof. To verify the openness of $\mathcal{H}_{C / k}^{d+g-1}$, we use the characterization of openness of subfunctors given in 23, Lemma 2.2.3. For a $k$-scheme $S$, let $\mathcal{L}$ be an invertible sheaf on $C \times_{k} S$. Then condition (2a) is satisfied by the definition of the functor. For condition (2b), let $L / K$ be a field extension, and let $i:$ Spec $K \rightarrow \operatorname{Spec} L$ be the associated morphism. Since $i$ is flat, it follows that $\left(\pi_{L}\right)_{*}\left(1_{C_{k}} \times i\right)^{*} \mathcal{L} \cong$ $i^{*}\left(\pi_{K}\right)_{*} \mathcal{L}$. Now the condition follows since $\left(\left(1_{C_{k}} \times i\right)^{*} \mathcal{L}\right)(-1) \cong\left(1_{C_{k}} \times i\right)^{*}(\mathcal{L}(-1))$. For condition (2c), we need to prove that the set of points $\{s\}$ in $S$ for which $\mathcal{L}_{s}$ is in $\mathcal{H}_{C / k}^{d+g-1}(\operatorname{Spec} k(s))$ is open in $S$. We note that the image of a point $s$ of $S$ in $\operatorname{Pic}_{C / k}^{d+g-1}$ lies in Spec $A_{k}$ if and only if $h^{0}\left(C_{s}, \mathcal{L}_{s}(-1)\right)=0$ (Proposition [2.6). Now the condition follows by noting that the set in question is the inverse image of Spec $A_{k}$ under the morphism $S \rightarrow \operatorname{Pic}_{C / k}^{d+g-1}$.

Moreover, by the discussion above, it also follows that the canonical morphism $S \rightarrow \operatorname{Pic}_{C / k}^{d+g-1}$ associated with an element of $\mathcal{H}_{C / k}^{d+g-1}(S)$ factors through Spec $A_{k}$. Note that the restriction of the universal invertible sheaf to $C \times_{k}$ Spec $A_{k}$ is an element of $\mathcal{H}_{C / k}^{d+g-1}\left(\operatorname{Spec} A_{k}\right)$. This follows from Proposition 2.6. This shows that Spec $A_{k}$ represents the functor $\mathcal{H}_{C / k}^{d+g-1}$.

We can now construct a natural transformation

$$
\Phi: \mathcal{G}_{d}\left(\tilde{C}_{f},-\right) \longrightarrow \mathcal{H}_{C / k}^{d+g-1}(-) .
$$

Namely, to any element $(\mathcal{E} n d(\mathcal{E}), \psi)$ in $\mathcal{G}_{d}\left(\tilde{C}_{f}, S\right)$, we assign the $\mathcal{O}_{C \times{ }_{k} S}$-module $\left(H^{0}(S, \mathcal{E}) \otimes_{\mathcal{O}_{S}} \mathcal{O}_{S}[u, v]\right)^{\sim}$. To see that $\Phi$ is a natural transformation, let $f: T \rightarrow S$ be a morphism of schemes and let $(\mathcal{E} n d(\mathcal{E}), \psi)$ be an element of $\mathcal{G}_{d}\left(\tilde{C}_{f}, S\right)$. Then we need to prove that the two invertible $\mathcal{O}_{C \times_{k} T}$-modules $\mathcal{O}_{T} \otimes_{\mathcal{O}_{S}} p_{S}^{*} \mathcal{E}$ and $p_{T}^{*} f^{*} \mathcal{E}$ are isomorphic. Now the statement is immediate, since the action of $w$ is preserved by the natural $\mathcal{O}_{\mathbb{P}_{T}^{1}}$-module isomorphism between these sheaves. 
Since the functor $\mathcal{H}_{C / k}^{d+g-1}$ is representable, by sheafification of $\Phi$, we get the natural transformation

$$
\Phi: \mathcal{R} e p_{d}\left(\tilde{C}_{f},-\right) \cong \overline{\mathcal{G}}_{d}\left(\tilde{C}_{f},-\right) \longrightarrow \mathcal{H}_{C / k}^{d+g-1}(-) .
$$

The isomorphism in the above definition follows from Lemma 5.1. Now the functors $\mathcal{R} e p_{d}\left(\tilde{C}_{f},-\right)$ and $\mathcal{H}_{C / k}^{d+g-1}(-)$ are represented by Spec $Z_{k}$ and Spec $A_{k}$, respectively. This follows from the fact that $\tilde{C}_{f}$ is an Azumaya algebra over its center and Lemma 5.4. So the natural transformation induces a morphism of schemes

$$
\eta: \operatorname{Spec} Z_{k} \longrightarrow \operatorname{Spec} A_{k} .
$$

In the following section, we will show that the morphisms $\eta$ and $\varphi$ are inverses of each other.

\section{THE ISOMORPHISM THEOREM AND DESCENT ON $\varphi$}

In this section, we first prove that the morphism $\varphi$ is an isomorphism. Now consider the composition

$$
\text { Spec } A_{k} \stackrel{\varphi}{\longrightarrow} \operatorname{Spec} Z_{k} \stackrel{\eta}{\longrightarrow} \operatorname{Spec} A_{k},
$$

and denote it by $\theta$. In the following proposition, $\mathcal{P}$ denotes the restriction of the universal invertible sheaf to $C \times{ }_{k}$ Spec $A_{k}$.

Proposition 6.1. The morphism $\theta$ is the identity morphism on Spec $A_{k}$.

Proof. Note that since $\mathcal{P}$ is the universal object in $\mathcal{H}_{C / k}^{d+g-1}$ (Spec $\left.A_{k}\right)$, it will be sufficient to prove that $\left(1_{C} \times \theta\right)^{*} \mathcal{P}$ is isomorphic to $\mathcal{P}$ as a rigidified line bundle. Furthermore, by Remark [2.15] it is sufficient to show that these two sheaves are isomorphic as line bundles. Note that the sheaf $\left(1_{C} \times \theta\right)^{*} \mathcal{P}$ is isomorphic to the image under $\Phi$ of $(\varphi, \mathcal{E} n d(\mathcal{P}))$ in $\mathcal{R e p}_{d}\left(\tilde{C}_{f}, S p e c A_{k}\right)$. But then the sheaf $\left(1_{C} \times \theta\right)^{*} \mathcal{P}$ is isomorphic to $\left(P \otimes_{A_{k}} A_{k}[u, v]\right)^{\sim}$, where $P=H^{0}\left(\operatorname{Spec} A_{k},\left(\pi_{A_{k}}\right)_{*}(\mathcal{P})\right)$ and $w$ acts as $u \varphi\left(x_{1}\right)+v \varphi\left(x_{2}\right)$. Now from the construction of the morphism $\varphi$ (Proposition 4.2), the two actions of $w$ on the graded $\left(A_{k}[u, v, w] /\left(w^{d}-f(u, v)\right)\right)$-module $P \otimes_{A_{k}} A_{k}[u, v]$ corresponding to the invertible sheaves $\left(1_{C} \times \theta\right)^{*} \mathcal{P}$ and $\mathcal{P}$ on $C \times{ }_{k}$ Spec $A_{k}$ are compatible. So the sheaves in question are isomorphic. This shows that $\theta$ is the identity morphism.

Now we can prove the following theorem.

Theorem 6.2. Let $k$ be a field so that the curve $C$ has a k-rational point. Then the morphism $\varphi$ is an isomorphism.

Proof. We prove that the corresponding homomorphism $\varphi: Z_{k} \rightarrow A_{k}$ is an isomorphism. By Proposition 4.8, it is sufficient to show that the map $\varphi$ is surjective. By Proposition 6.1, there exists a ring homomorphism $\eta: A_{k} \rightarrow Z_{k}$ so that the composition

$$
A_{k} \stackrel{\eta}{\longrightarrow} Z_{k} \stackrel{\varphi}{\longrightarrow} A_{k}
$$

is the identity homomorphism. In particular, $\varphi$ is surjective. It follows that $\varphi$ is an isomorphism. 
6.1. The Galois descent on $\varphi$. In this section, we want to prove the isomorphism theorem of the last section without the assumption that the curve $C$ has a $k$-rational point. The idea is to prove that the $\varphi$ obtained earlier descends to a field over which $f$ is defined.

Let $f$ be a binary form over a field $k$ such that the characteristic of $k$ does not divide $d$ and $f$ does not have repeated factors over an algebraic closure of $k$. Let $k^{\prime} / k$ be a finite Galois extension (with Galois group $G$ ) such that $C\left(k^{\prime}\right)$ is nonempty. Here we may assume that $k^{\prime}$ is a Galois extension of $k$ by the assumption on $\operatorname{char}(k)$. Then we have the isomorphism

$$
\varphi: \operatorname{Spec} A_{k^{\prime}} \longrightarrow \operatorname{Spec} Z_{k^{\prime}} .
$$

Recall that $S p e c A_{k^{\prime}}$ descends to $\operatorname{Spec} A_{k}$, which is the complement of the $\Theta$-divisor in $\operatorname{Pic}_{C / k}^{d+g-1}$. Also, Spec $Z_{k^{\prime}}$ descends to $S p e c Z_{k}$, which is the center of the Clifford algebra of $f$ over $k$.

Main Theorem. The morphism $\varphi$ descends to $k$, and hence the center of the reduced Clifford algebra is isomorphic to the coordinate ring of the complement of the $\Theta$-divisor in $\mathrm{Pic}_{C / k}^{d+g-1}$.

Proof. Let $x$ be a closed point of Spec $A_{k^{\prime}}$, and let $k(x)$ be its residue field. Let $\sigma \in G$. Then we have the following diagram:

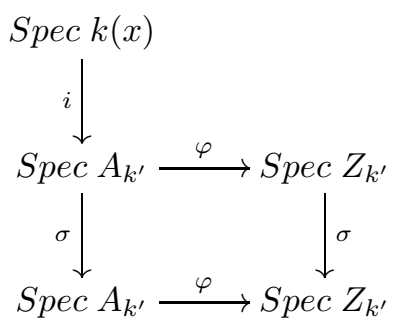

Now we prove that the above diagram is commutative. Let $j$ denote the open immersion Spec $A_{k^{\prime}} \hookrightarrow \operatorname{Pic}_{C^{\prime} / k^{\prime}}^{d+g-1}$. We first show that

$$
j \circ \sigma \circ i=j \circ \varphi^{-1} \circ \sigma \circ \varphi \circ i .
$$

Let $\pi$ denote the structure morphism $\operatorname{Pic}_{C^{\prime} / k^{\prime}}^{d+g-1} \rightarrow$ Spec $k^{\prime}$, and $C^{\prime} \times_{k^{\prime}} k(x)^{\sigma}$ the fibred product of $C^{\prime}$ and Spec $k(x)$ with the structure morphism for Spec $k(x)$ as $\pi \circ \sigma \circ j \circ i$. Also, let $C^{\prime} \times_{k^{\prime}} k(x)$ denote the fibred product as earlier with the structure morphism for Spec $k(x)$ as $\pi \circ j \circ i$. We have the isomorphism

$$
C^{\prime} \times_{k^{\prime}} k(x) \stackrel{\sigma \times \mathrm{id}}{\longrightarrow} C^{\prime} \times_{k^{\prime}} k(x)^{\sigma} .
$$

To prove (6.1), it is sufficient to prove that the line bundles on $C^{\prime} \times_{k^{\prime}} k(x)^{\sigma}$ corresponding to these morphisms are isomorphic. See Lemma 2.13. If $\mathcal{P}$ denotes the universal line bundle on $C^{\prime} \times{ }_{k^{\prime}} \mathrm{Pic}_{C^{\prime} / k^{\prime}}^{d+g-1}$, then the line bundle corresponding to the morphism on the left side of (6.1) is $(\mathrm{id} \times(j \circ \sigma \circ i))^{*} \mathcal{P}$.

Consider the morphism on the right side of the above equation. To construct the corresponding line bundle, it is sufficient to consider the associated representation of the Clifford algebra. The required line bundle corresponds to the following composite morphism:

$$
\tilde{C}_{f} \otimes_{k} k^{\prime} \stackrel{\operatorname{id} \times \sigma}{\longrightarrow} \tilde{C}_{f} \otimes_{k} k^{\prime} \stackrel{\eta_{i}}{\longrightarrow} M_{d}(k(x)),
$$


where $\eta_{i}$ is the representation corresponding to the morphism $i$ (or $i \circ \varphi$ ). But this line bundle is isomorphic to the pull-back of $\mathcal{P}$ under the composite morphism

$$
C^{\prime} \times_{k^{\prime}} k(x)^{\sigma} \stackrel{\sigma^{-1} \times \text { id }}{\longrightarrow} C^{\prime} \times_{k^{\prime}} k(x) \stackrel{\text { id } \times(j \circ i)}{\longrightarrow} C^{\prime} \times_{k^{\prime}} \operatorname{Pic}_{C^{\prime} / k^{\prime}}^{d+g-1} .
$$

Now consider the commutative diagram

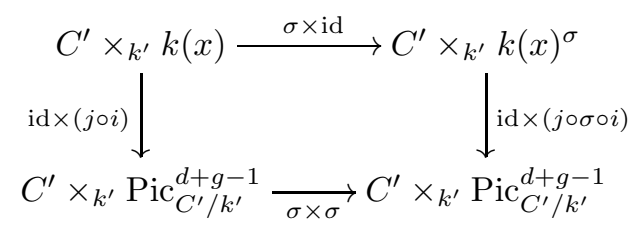

By the above argument and the commutativity of this diagram, it follows that the two line bundles in question are isomorphic to

$$
(\operatorname{id} \times(j \circ \sigma \circ i))^{*} \mathcal{P} \quad \text { and } \quad(\operatorname{id} \times(j \circ \sigma \circ i))^{*}\left(\sigma^{-1} \times \sigma^{-1}\right)^{*} \mathcal{P} .
$$

By Lemma 2.12 these two line bundles are isomorphic. So (6.1) holds true.

In particular, $i=\left(\sigma^{-1} \circ \varphi^{-1} \circ \sigma \circ \varphi\right) \circ i$. Now consider the two automorphisms id and $\mu=\left(\sigma^{-1} \circ \varphi^{-1} \circ \sigma \circ \varphi\right)$ of Spec $A_{k^{\prime}}$. They both are the identity on closed points, and the morphisms induced on the residue fields are the same. Let $a \in A_{k^{\prime}}$. Then $(\mu(a)-a)$ is in any maximal ideal on $A_{k^{\prime}}$. Since $A_{k^{\prime}}$ is of finite type over $k^{\prime}$ and is an integral domain, the intersection of all the maximal ideals is the zero ideal. So $\mu(a)=a$ for any $a \in A_{k^{\prime}}$. This shows that $\mu$ is the identity morphism. 2.7.

The second part follows from the discussion preceding the theorem and Corollary

We record an easy corollary of the main theorem.

Corollary 6.3. The center $Z_{k}$ of the reduced Clifford algebra is an integrally closed Noetherian domain.

Proof. By the main theorem, the $k$-scheme $S p e c Z_{k}$ is isomorphic to the scheme Spec $A_{k}$, which is the complement of the $\Theta$-divisor in $\operatorname{Pic}_{C / k}^{d+g-1}$. Since $\operatorname{Pic}_{C / k}^{d+g-1}$ is a smooth, integral Noetherian scheme and Spec $A_{k}$ is an open subscheme of $\operatorname{Pic}_{C / k}^{d+g-1}$, Spec $A_{k}$ has these properties as well. In particular, $A_{k}$ is an integrally closed Noetherian domain. So $Z_{k}$ is an integrally closed Noetherian domain.

Remark 6.4. The Main Theorem6.1 says that the reduced Clifford algebra can be viewed as a sheaf of Azumaya algebras on Spec $A_{k}$. There are two natural questions which arise. The first is, does this sheaf extend to $\mathrm{Pic}_{C / k}^{d+g-1}$ as an Azumaya algebra? The second is if it does, then what is its Brauer class? These questions will be addressed in a forthcoming article [13.

\section{ACKNOWLEDGEMENTS}

We thank Darrell Haile, Michael Larsen and Valery Lunts for their help and encouragement. This project has benefitted immensely from our conversations with them and from their advice. 


\section{REFERENCES}

1. E. Arbarello, M. Cornalba, P. A. Griffiths and J. Harris, Geometry of Algebraic Curves, vol. 1, Springer-Verlag, New York, 1985. MR 86h:14019

2. S. Bosch, W. Lütkebohmert and M. Raynaud, Néron Models, Springer-Verlag, New York, 1990. MR 91i:14034

3. L. Childs, Linearizing of $n$-ic forms and generalized Clifford algebras, Linear and Multilinear Algebra 5 (1978), 267-278. MR 57:12567.

4. F. DeMeyer and E. Ingraham, Separable Algebras over Commutative Rings, Lecture Notes in Math., vol. 181, Springer-Verlag, Berlin, 1971. MR 43:6199

5. W. Fulton, Intersection Theory, Springer-Verlag, New York, 1998. MR 99d:14003

6. A. Grothendieck and J. Dieudonné, Eléments de Géométrie Algébrique, Inst. Hautes Études Sci. Publ. Math., nos. 4, 8, 11, 17, 20, 24, 28, 32 (1964-1967). MR 29:1210 MR 30:3885 MR 33:7330 MR 36:178 MR 39:220

7. A. Grothendieck, Technique de descente et théorèmes d'existence en géométrie algébrique. II: Le théorème d'existence en théorie formelle des modules, Séminaire Bourbaki 1959/60, Exposé 195, Secrétariat Math., Paris, 1960 (and later reprints by other publishers). MR 23:A2273

8. D. Haile, On the Clifford algebra of a binary cubic form, Amer. J. Math. 106 (1984), 12691280. MR 86c: 11028

9. D. Haile, When is the Clifford algebra of a binary cubic form split, J. Algebra 146 (1992), 514-520. MR 93a:11029

10. D. Haile and S. Tesser, On Azumaya algebras arising from Clifford algebras, J. Algebra 116 (1988), 372-384. MR 89j:15044

11. R. Hartshorne, Algebraic Geometry, Springer-Verlag, 1977. MR 57:3116

12. R. S. Kulkarni, On the Clifford algebra of a binary form, Ph.D. Thesis, Indiana University, 1999.

13. R. S. Kulkarni, On the extension of the Brauer class of the reduced Clifford algebra, submitted.

14. J. Milne, Abelian Varieties: Arithmetic Geometry, Springer-Verlag, 1986, pp. 103-150. MR 89b:14029

15. J. Milne, Jacobian Varieties: Arithmetic Geometry, Springer-Verlag, 1986, pp. 167-212. MR 89b:14029

16. J. Milne, Étale Cohomology, Princeton University Press, 1980. MR 81j:14002

17. S. Montgomery and L. W. Small, Fixed rings of Noetherian rings, Bull. London Math. Soc. 13 (1981), 33-38. MR 82a:16033

18. D. Mumford, Abelian Varieties, 2nd ed., Oxford University Press, 1970, MR 44:219

19. C. Processi, Rings with Polynomial Identities, Marcel Dekker, New York, 1973. MR 51:3214

20. P. Revoy, Algèbres de Clifford et algèbres extérieures, J. Algebra 46 (1977), 268-277. MR 57:12568

21. N. Roby, Algèbres de Clifford des formes polynômes, C. R. Acad. Sci. Paris Sér. I Math. A-B 268 (1969), A484-A486. MR 39:2794

22. M. Van den Bergh, Linearisations of binary and ternary forms, J. Algebra 109 (1987), 172-183. MR 88j:11020

23. M. Van den Bergh, The center of the generic division algebra, J. Algebra 127 (1989), 106-126. MR 91d:16031

Department of Mathematics, University of Wisconsin-Madison, Madison, Wisconsin 53706

Current address: Department of Mathematics, Wells Hall, Michigan State University, East Lansing, Michigan 48824

E-mail address: kulkarni@math.msu.edu 